\title{
How do Black Holes Spin in Chern-Simons Modified Gravity?
}

\author{
Daniel Grumiller ${ }^{1}$ and Nicolás Yunes ${ }^{2}$ \\ ${ }^{1}$ Center for Theoretical Physics, Massachusetts Institute of Technology, \\ 77 Massachusetts Ave., Cambridge, MA 02139, USA \\ ${ }^{2}$ Institute for Gravitation and the Cosmos, Department of Physics, \\ The Pennsylvania State University, University Park, PA 16802, USA
}

(Dated: October 25, 2018)

\begin{abstract}
No Kerr-like exact solution has yet been found in Chern-Simons modified gravity. Intrigued by this absence, we study stationary and axisymmetric metrics that could represent the exterior field of spinning black holes. For the standard choice of the background scalar, the modified field equations decouple into the Einstein equations and additional constraints. These constraints eliminate essentially all solutions except for Schwarzschild. For non-canonical choices of the background scalar, we find several exact solutions of the modified field equations, including mathematical black holes and pp-waves. We show that the ultrarelativistically boosted Kerr metric can satisfy the modified field equations, and we argue that physical spinning black holes may exist in Chern-Simons modified gravity only if the metric breaks stationarity, axisymmetry or energy-momentum conservation.
\end{abstract}

PACS numbers: 04.20.Cv,04.70.Bw,04.20.Jb,04.30.-w

\section{INTRODUCTION}

General relativity (GR) is one of physics' most successful theories, passing all experimental tests so far with ever increasing accuracy [1]. Nevertheless, modifications to GR are pursued vigorously for two main reasons: from a theoretical standpoint, we search for an ultraviolet (UV) completion of GR, such as string theory, that would lead to corrections in the action proportional to higher powers of scalar invariants of the Riemann tensor; from an experimental standpoint, observations in the deep infrared (IR) regime suggest the existence of some form of dark energy [2, 3, 4]. One possibility to accommodate dark energy is to consider an action with non-linear couplings to the Ricci scalar [5, 6], similar in spirit to the corrections that we expect from a UV completion of GR.

UV and IR corrections entail higher derivatives of the fundamental degrees of freedom in the equations of motion, which on general grounds tend to have disastrous consequences on the stability of the solutions of the theory [87]: the so-called Ostrogradski instability (for a review cf. e.g. [7]). A few loopholes exist, however, that allow to bypass this theorem (for example, if the nonlinear corrections can be converted into a representation of a scalar-tensor theory). Along these lines, special combinations of scalar invariants that play the role of a topological term, such as the Euler or Pontryagin term, can in general be added safely to the action.

In this paper, we study Chern-Simons (CS) modified gravity [8], where the Einstein-Hilbert action is modified by the addition of a parity-violating Pontryagin term. As described by Jackiw and Pi [8], this correction arises through the embedding of the 3-dimensional CS topological current into a 4-dimensional spacetime manifold. CS gravity is not a random extension of GR, but it has physical roots in particle physics. Namely, if there is an imbalance between left- $\left(N_{L}\right)$ and right-handed $\left(N_{R}\right)$ fermions, then the fermion number current $j^{\mu}$ has a well-known gravitational anomaly $[9], \partial_{\mu} j^{\mu} \propto\left(N_{L}-N_{R}\right)^{*} R R$, analogous to the original triangle anomaly [10]. Here ${ }^{*} R R$ is the Pontryagin term (also known as the gravitational instanton density or Chern-Pontryagin term) to be defined in the next section. CS gravity is also motivated by string theory: it emerges as an anomaly-canceling term through the Green-Schwarz mechanism [11]. Such a correction to the action is indispensable, since it arises as a requirement of all 4-dimensional compactifications of string theory in order to preserve unitarity [12].

CS gravity has been studied in the context of cosmology, gravitational waves, solar system tests and Lorentz invariance. In particular, this framework has been used to explain the anisotropies in the cosmic microwave background [13, 14, 15] and the leptogenesis problem [12, 16] (essentially using the gravitational anomaly described above in the other direction). CS gravity has also been shown to lead to amplitude birefringent gravitational waves [8, 17, 18, 19], possibly allowing for a test of this theory with gravitational-wave detectors [20]. Moreover, CS gravity has been investigated in the far-field of a spinning binary system, leading to a prediction of gyromagnetic precession [18, 19] that differs from GR. This prediction was later improved on and led to a constraint on the magnitude of the CS coupling [21]. Finally, CS gravity has been studied in the context of Lorentz-invariance and -violation 22] and the theory has been found to preserve this symmetry, provided the CS coupling is treated as a dynamical field. For further studies of these and related issues cf. e.g. 8, 15, 17, 19, 21, 22, 23, 24, 25, 26, $27,28,29,30,31,32,33,34,35$.

CS gravity introduces the following modification to the action [8]: $S=S_{E H}+S_{\text {mat }}+S_{C S}$, where $S_{E H}$ is the Einstein-Hilbert action, $S_{\text {mat }}$ is some matter action, and the new term is given by

$$
S_{C S} \sim \int d V \theta^{*} R R
$$


In Eq. (1), $d V$ is a 4 -dimensional volume element, ${ }^{*} R R$ is the Pontryagin term and $\theta$ is a background scalar field (we shall define this action in more detail in the next section). This scalar field, sometimes called a gravitational axion, acts as a CS coupling function that can be interpreted either as an external or a dynamical quantity. In the former case, CS gravity is an effective theory that derives from some other, more fundamental gravity theory that physically defines the scalar field. In the latter case, the scalar field possesses its own equation of motion, which could in principle contain a potential and a kinetic term [21].

The strength of the CS correction clearly depends on the CS coupling function. If we consider CS gravity as an effective theory, the coupling function is suppressed by some mass scale, which could lie between the electro-weak and the Planck scale, but it is mostly unconstrained [21]. In the context of string theory, the coupling constant has been computed in very conservative scenarios, leading to a Planck mass suppression [16]. In less conservative scenarios, there could exist enhancements that elevate the coupling function to the realm of the observable. Some of these scenarios are cosmologies where the string coupling vanishes at late times [36, 37, 38, 39, 40, 41, 42, 43, 44, 45, 46], or where the field that generates $\theta$ couples to spacetime regions with large curvature [47, 48] or stress-energy density [20].

The CS correction is encoded in the modified field equations, which can be obtained by varying the modified action with respect to the metric. The divergence of the modified field equations establishes the Pontryagin constraint ${ }^{*} R R=0$, through the Bianchi identities for a vacuum or conserved stress-energy tensor. Not only does this constraint have important consequences on the conservation of energy, but it also restricts the space of solutions of the modified theory. For example, although this restriction is not strong enough to eliminate the Schwarzschild solution, it does eliminate the Kerr solution. Since astrophysical observations suggest that supermassive black holes (BHs) at the center of galaxies do have a substantial spin (cf., e. g. [49] and references therein), this raises the interesting question of what replaces the Kerr solution in CS gravity.

In this paper, we search for solutions to the CS modified field equations that could represent the exterior gravitational field of a spinning star or BH. We find that solutions cluster into two different classes: GR solutions that independently satisfy both the vacuum Einstein equations and the modified field equations; non-GR solutions that satisfy the modified field equations but not the vacuum Einstein equations. We carry out an extensive study of solutions by looking at three groups of line elements: spherically symmetric metrics; static and axisymmetric metrics; and stationary and axisymmetric metrics. The first group contains GR solutions only, independently of the choice of the CS scalar field. The second group leads to a decoupling of the modified field equations for 'natural' choices of the scalar field, which again reduces to trivial GR solutions. In fact, we show here that static and axisymmetric line elements are forced to be spatially conformally flat if such a decoupling occurs. The third group also leads to the same decoupling for the canonical choice of the scalar field, and we argue against the existence of non-trivial solutions.

This paper suggests that stationary and axisymmetric line elements in CS gravity probably do not admit solutions of the field equations for the canonical choice of the CS scalar field. However, solutions do exist when more general scalar fields are considered, albeit not representing physical $\mathrm{BH}$ configurations [88]. We find two types of solutions, mathematical BHs and ultrarelativistically boosted $\mathrm{BHs}$, which, to our knowledge, are the first examples of BH and BH-like solutions in CS gravity, besides Schwarzschild and Reissner-Nordström. The first type arises when we consider a subclass of stationary and axisymmetric line elements (the so-called van Stockum class), for which we find both GR and non-GR solutions for non-canonical scalar fields. For instance, we shall demonstrate that the line-element

$$
d s^{2}=-\rho\left(1-\frac{2 m}{\sqrt{\rho}}\right) d t^{2}-2 \rho d t d \phi+\frac{1}{\sqrt{\rho}}\left(d \rho^{2}+d z^{2}\right),
$$

together with the CS scalar field $\theta=2 \sqrt{\rho} z / 3$, satisfies the modified field equations but does not arise in GR as a vacuum solution. The metric in Eq. (2) represents BHs in the mathematical sense only: it exhibits a Killing horizon at $\sqrt{\rho}=2 m=$ const., but it contains unphysical features, such as (naked) closed time-like curves. The second type of solutions with a non-canonical scalar field arises when we consider scalar fields whose divergence is a Killing vector. These fields lead to exact gravitational pp-wave solutions of GR and non-GR type. One particular example that we shall discuss in in this paper is the ultrarelativistically boosted Kerr BH,

$$
d s^{2}=-2 d u d v-h_{0} \delta(u) \ln \left(x^{2}+y^{2}\right) d u^{2}+d x^{2}+d y^{2},
$$

with the CS scalar field $\theta=\lambda v$, where $h_{0}$ and $\lambda$ are constants.

Although we did not find a Kerr analogue by searching for stationary and axisymmetric solutions, spinning BHs do seem to exist in the theory. This suggestion is fueled by the existence of two different limits of the Kerr spacetime that are still preserved: the Schwarzschild limit and the Aichelburg-Sexl limit, Eq. (3), which we shall show persists in CS gravity. These limits, together with the existence of a non-axisymmetric far-field solution [19], indicate that a spinning $\mathrm{BH}$ solution must exist, albeit not with the standard symmetries of the Kerr spacetime. Unfortunately, spacetimes with only one or no Killing vector are prohibitively general and their study goes beyond the scope of this work. Nonetheless, the possibility of constructing such solutions by breaking stationarity or axisymmetry is discussed and a better understanding of solutions in CS gravity is developed. Finally, we show how to recover the Kerr solution by postulating, in 
an ad-hoc manner, a non-conserved energy momentumtensor and deduce that it violates the classical energy conditions.

This paper is organized as follows: Sec. [1]reviews some basic features of CS modified gravity and exploits two alternative formulations of the Pontryagin constraint, one based upon the spinorial decomposition of the Weyl tensor and one based upon its electro-magnetic decomposition, to reveal some physical consequences of this constraint; Sec. III revisits the Schwarzschild, FriedmannRobertson-Walker and Reissner-Nordström solutions in CS modified gravity and addresses the sensitivity of these solutions to the choice of CS coupling function; Sec. IV studies static, axisymmetric line elements in great detail, while Sec. $\mathrm{V}$ investigates stationary, axisymmetric metrics and provides the first non-trivial exact solutions to CS modified gravity, including mathematical $\mathrm{BH}$ solutions; Sec. VI addresses metrics that break axisymmetry or stationarity and concentrates on non-trivial solutions for pp-waves and the Aichelburg-Sexl boosted Kerr metric; Sec. VII concludes and points to future research.

We use the following conventions in this paper: we work exclusively in four spacetime dimensions with signature $(-,+,+,+)$ [50], with Latin letters $(a, b, \ldots, h)$ ranging over all spacetime indices; curvature quantities are defined as given in the MAPLE GRTensorII package [51]; round and square brackets around indices denote symmetrization and anti-symmetrization respectively, namely $T_{(a b)}:=\frac{1}{2}\left(T_{a b}+T_{b a}\right)$ and $T_{[a b]}:=\frac{1}{2}\left(T_{a b}-\right.$ $\left.T_{b a}\right)$; partial derivatives are sometimes denoted by commas, e.g. $\partial \theta / \partial r=\partial_{r} \theta=\theta_{, r}$. The Einstein summation convention is employed unless otherwise specified, and we use geometrized units where $G=c=1$.

\section{CS MODIFIED GRAVITY}

\section{A. $\mathrm{ABC}$ of $\mathrm{CS}$}

In this section, we summarize the basics of CS modified gravity, following the formulation of [8]. Let us begin by defining the full action of the theory [89]:

$$
S=\kappa \int d^{4} x \sqrt{-g}\left(R-\frac{1}{4} \theta{ }^{*} R R\right)+S_{\mathrm{mat}},
$$

where $\kappa=1 /(16 \pi), g$ is the determinant of the metric, the integral extends over all spacetime, $R$ is the Ricci scalar, $S_{\text {mat }}$ is some unspecified matter action and ${ }^{*} R R$ is the Pontryagin term. The latter is defined via

$$
{ }^{*} R R:={ }^{*} R^{a}{ }_{b}{ }^{c d} R^{b}{ }_{a c d},
$$

where the dual Riemann-tensor is given by

$$
{ }^{*} R_{b}^{a}{ }^{c d}:=\frac{1}{2} \epsilon^{c d e f} R_{b e f}^{a},
$$

with $\epsilon^{\text {cdef }}$ the 4-dimensional Levi-Civita tensor [90]. The Pontryagin term [Eq. (50)] can be expressed as the diver- gence

$$
\nabla_{a} K^{a}=\frac{1}{4}{ }^{*} R R
$$

of the Chern-Simons topological current $(\Gamma$ is the Christoffel connection),

$$
K^{a}:=\epsilon^{a b c d}\left(\Gamma_{b m}^{n} \partial_{c} \Gamma_{d n}^{m}+\frac{2}{3} \Gamma_{b m}^{n} \Gamma_{c l}^{m} \Gamma_{d n}^{l}\right),
$$

thus the name "Chern-Simons modified gravity" 91].

The modified field equations can be obtained by varying the action with respect to the metric. Exploiting the well-known relations

$$
\delta R_{a c d}^{b}=\nabla_{c} \delta \Gamma_{a d}^{b}-\nabla_{d} \delta \Gamma_{a c}^{b}
$$

and

$$
\delta \Gamma_{a c}^{b}=\frac{1}{2} g^{b d}\left(\nabla_{a} \delta g_{d c}+\nabla_{c} \delta g_{a d}-\nabla_{d} \delta g_{a c}\right),
$$

the variation of the geometric part of the action leads to

$$
\begin{aligned}
\delta S-\delta S_{\mathrm{mat}} & =\kappa \int d^{4} x \sqrt{-g}\left(R_{a b}-\frac{1}{2} g_{a b} R+C_{a b}\right) \delta g^{a b} \\
& -\frac{\kappa}{4} \int d^{4} x \sqrt{-g}^{*} R R \delta \theta \\
& +\Sigma_{E H}+\Sigma_{C S} .
\end{aligned}
$$

Here, the tensor $C_{a b}$ stands for a 4-dimensional Cottonlike tensor, which we shall refer to as the C-tensor [92], given by

$$
C^{a b}:=v_{c} \epsilon^{c d e(a} \nabla_{e} R_{d}^{b)}+v_{c d}{ }^{*} R^{d(a b) c},
$$

where

$$
v_{a}:=\nabla_{a} \theta, \quad v_{a b}:=\nabla_{a} \nabla_{b} \theta=\nabla_{(a} \nabla_{b)} \theta
$$

are the velocity and covariant acceleration of $\theta$, respectively [93]. We shall always assume that $v_{a}$ does not vanish identically, because otherwise the model reduces to GR [94].

Surface terms are collected in the third line of Eq. (11) and arise due to repeated integration by parts and application of Stokes' theorem. In particular, $\Sigma_{E H}$ and $\Sigma_{C S}$ arise from variation of the Einstein-Hilbert and CS sector of the action, respectively. The former expression is well-known, while the latter contains a term with $\delta \Gamma$,

$$
\Sigma_{C S}=\kappa \int d^{4} x \sqrt{-g} \nabla_{d}\left(\theta^{*} R^{a b c d} \delta \Gamma_{b a c}\right)+\ldots
$$

It is worthwhile pointing out that one cannot just impose Dirichlet boundary conditions on the induced metric at the boundary by adding the Gibbons-Hawking-York term, as it is the case in GR [52, 53]. There is no obvious way to cancel the term containing the variation of the connection, $\delta \Gamma$, in Eq. (14), except by imposing suitable 
fall-off conditions on the scalar field $\theta$ or Dirichlet boundary conditions on the connection. Even though we shall neglect boundary issues henceforth, we emphasize that these considerations are relevant in many applications, such as BH thermodynamics.

The modified field equations are then given by the first line of Eq. (11), provided the second line vanishes. The vanishing of ${ }^{*} R R$ is the so-called Pontryagin constraint and we shall study it in Sec. IIB The modified field equations in the presence of matter sources are then given by

$$
G_{a b}+C_{a b}=8 \pi T_{a b},
$$

where $G_{a b}=R_{a b}-\frac{1}{2} g_{a b} R$ is the Einstein tensor and $T_{a b}$ is the stress-energy tensor of the source. In this paper, we are primarily concerned with the vacuum case, $T_{a b}=0$, for which the modified field equations reduce to

$$
R_{a b}+C_{a b}=0
$$

due to the tracelessness of the C-tensor, $C^{a}{ }_{a}=0$. Like in GR, vacuum solutions in CS gravity satisfy

$$
R=0
$$

\section{B. Pontryagin Constraint}

Let us now discuss the Pontryagin constraint

$$
{ }^{*} R R=0
$$

which then forces the second line in Eq. (11) to vanish. One route to obtain the Pontryagin constraint is to treat $\theta$ as a dynamical field (or rather a Lagrange multiplier). By varying the action with respect to $\theta$, we obtain the equations of motion for the scalar field that dynamically enforce the Pontryagin constraint.

Another route to obtain the Pontryagin constraint is to treat $\theta$ as an external quantity. In this case, there are no equations of motion for the scalar field. Nonetheless, by taking the covariant divergence of the equations of motion and using the contracted Bianchi identities, one obtains

$$
\nabla_{a} C^{a b}=\frac{1}{8} v^{b *} R R=8 \pi \nabla_{a} T^{a b}
$$

Usually, it is desirable to require that the stress-energy be covariantly conserved. However, in CS modified gravity this need not be the case because a non-vanishing covariant divergence $\nabla_{a} T^{a b} \neq 0$ could be balanced by a non-vanishing Pontryagin term - this is, in fact, how the term arises in some approaches in the first place, cf. [27]. We shall come back to this issue at the end of Sec. VI. but for the time being we shall set $T^{a b}=0$, which then leads to the Pontryagin constraint.

The Pontryagin constraint is a necessary condition for any vacuum spacetime that solves the modified field equations, but what does it mean physically? We shall attempt to answer this question by providing two alternative formulations of this constraint, but before doing so, let us discuss some general properties and consequences of Eq. (18). First, notice that setting the ${ }^{*} R R$ term to zero leads to the conserved current $K^{a}$ [Eqs. (7) and (8)], which is topological in nature, and thus implies this quantity is intrinsically different from typical conserved quantities, such as energy or angular momentum. Second, when the CS action is studied on-shell [Eq. (4) with ${ }^{*} R R=0$ ] it reduces to the GR action, an issue that is of relevance for stability considerations, e.g. thermodynamic stability in $\mathrm{BH}$ mechanics.

The first physical interpretation of the Pontryagin constraint can be obtained by considering a spinorial decomposition. Let us then consider the useful relation

$$
{ }^{*} R R={ }^{*} C C,
$$

which we prove in appendix A, In Eq. (20), $C$ is the Weyl tensor defined in $\mathrm{A2}$ and ${ }^{*} C$ its dual, defined in (A3). This identity allows us to use powerful spinorial methods to map the Weyl tensor into the Weyl spinor [54], which in turn can be characterized by the NewmanPenrose (NP) scalars $\left(\Psi_{0}, \Psi_{1}, \Psi_{2}, \Psi_{3}, \Psi_{4}\right)$. In the notation of [55], the Pontryagin constraint translates into a reality condition on a quadratic invariant of the Weyl spinor, $\mathcal{I}$,

$$
\Im(\mathcal{I})=\Im\left(\Psi_{0} \Psi_{4}+3 \Psi_{2}^{2}-3 \Psi_{1} \Psi_{3}\right)=0 .
$$

Such a reality condition is particularly useful for the consideration of algebraically special spacetimes. For instance, it follows immediately from Eq. (21) that spacetimes of Petrov types $I I I, N$ and $O$ obey the Pontryagin constraint, since in the latter case all NP scalars vanish, while in the former cases (in an adapted frame) only $\Psi_{3}$ or $\Psi_{4}$ are non-vanishing. Moreover, all spacetimes of Petrov types $D, I I$ and $I$ are capable of violating Eq. (21). For example, for spacetimes of Petrov type $I I$ one can choose an adapted tetrad such that $\Psi_{0}=\Psi_{1}=\Psi_{3}=0$, which then reduces Eq. (21) to the condition that either the real part or the imaginary part of $\Psi_{2}$ has to vanish.

The reality condition of Eq. (21) can also be useful in applications of $\mathrm{BH}$ perturbation theory. For instance, in the metric reconstruction of the perturbed Kerr spacetime [56], the NP scalars $\Psi_{1}=\Psi_{3}=0$ vanish. In this context gravitational waves are characterized by $\Psi_{4,0}$, while $\Psi_{2}$ is in general non-vanishing. In a tetrad that represents a transverse-traceless frame, these scalars are given by

$$
\Psi_{4,0}=\ddot{h}_{+} \mp i \ddot{h}_{\times}
$$

where $h_{+, \times}$are the plus/cross polarization of the waveform, and the overhead dot stands for partial time derivative [57]. Obviously, $\Psi_{0} \Psi_{4}=\left(\ddot{h_{+}}\right)^{2}+\left(\ddot{h_{\times}}\right)^{2}$ is real, which again reduces Eq. (21) to the condition that either the 
real part or the imaginary part of $\Psi_{2}$ has to vanish. Neither of these possibilities is the case for the Kerr $\mathrm{BH}$ or perturbations of it 56.

Another interpretation of the Pontryagin constraint can be obtained by exploiting the split of the Weyl tensor into electric and magnetic parts (cf. e.g. [58]). Given some time-like vector field $u^{a}$, normalized so that $u_{a} u^{a}=-1$, one can define the electric and magnetic parts of the Weyl tensor as

$$
\left(C_{a b c d}+\frac{i}{2} \epsilon_{a b e f} C_{c d}^{e f}\right) u^{b} u^{d}=E_{a c}+i B_{a c} .
$$

Then, the Pontryagin constraint is equivalent to the condition [95]

$$
E_{a b} B^{a b}=0 .
$$

This leads to three possibilities: either the spacetime is purely electric $\left(B_{a b}=0\right)$ or purely magnetic $\left(E_{a b}=0\right)$ or orthogonal, in the sense that Eq. (24) holds. Equation (24) is a perfect analogue to the condition ${ }^{*} F F \propto$ $\boldsymbol{E} \cdot \boldsymbol{B}=0$, which holds for specific configurations in electro-dynamics, including purely electric $(\boldsymbol{B}=0)$, purely magnetic $(\boldsymbol{E}=0)$ and electromagnetic wave configurations $(\boldsymbol{E} \neq 0 \neq \boldsymbol{B}, \boldsymbol{E} \cdot \boldsymbol{B}=0)$. This suggests that there could be single shock-wave solutions in CS gravity compatible with Eq. (24), which we shall indeed encounter in Sec. VI. In light of this electro-magnetic analogy, the Pontryagin constraint can be rephrased as "the gravitational instanton density must vanish," since the quantity ${ }^{*} F F$ is sometimes referred to as the "instanton density."

The electromagnetic decomposition of the Pontryagin constraint also allows for a physical interpretation in terms of perturbations of the Schwarzschild solution. In BH perturbation theory (cf. e.g. [59]), the metric perturbation is also decomposed through the electromagnetic Weyl tensor. The electric and magnetic parts can then be related to the flux of mass and angular momentum across the horizon. Suffice it to say that for a binary BH system in the slow-motion/small-hole approximation [59], these tensors are of order $E_{a b} \sim \mathcal{O}(\Phi)$ and $B_{a b} \sim \mathcal{O}(v \Phi)$, where the Newtonian potential $\Phi$ is of $\mathcal{O}\left(v^{2}\right)$ via the Virial theorem, with $v \ll 1$ the orbital velocity. In this case, the Pontryagin constraint is satisfied automatically up to terms of $\mathcal{O}\left(v^{5}\right)$. Within the post-Newtonian (PN) approximation [60], these conclusions imply that the PN metric for non-spinning pointparticles in the quasi-circular approximation violates the Pontryagin constraint at 2.5 PN order $\left[\mathcal{O}(v / c)^{5}\right]$, which is precisely the order at which gravitational waves appear.

Even for non-canonical choices of the scalar field, such as $\theta=z$ proposed in [33], the far field expansion of the Kerr metric does not satisfy the Pontryagin constraint to all orders. This is so because obviously ${ }^{*} R R$ is independent of $\theta$. In fact, one can show that violations of the constraint for the metric considered in [33] occur already at second order in the metric perturbation, which renders this metric hopeless as an exact CS solution. This

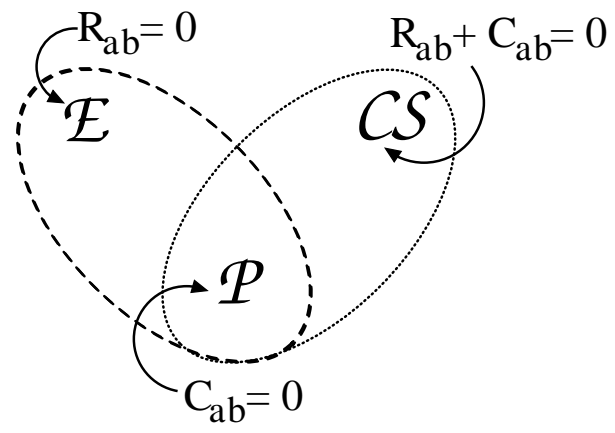

FIG. 1: Space of solutions of Einstein gravity $\mathcal{E}$ and CS modified gravity $\mathcal{C S}$.

observation is concurrent with the role the Pontryagin constraint may play for gravitational waves [61].

Finally, we can employ the electromagnetic analogy to anticipate the answer to the question we pose in the title of this paper. Namely, we are looking for a "rotating charge" configuration (where $\boldsymbol{E} \neq 0 \neq \boldsymbol{B}$ ), which simultaneously is an "electromagnetic wave" configuration (where $\boldsymbol{E} \cdot \boldsymbol{B}=0$ ). We know that no such solutions exist in electrodynamics, except for two limits [96]: if the rotation (and thus $\boldsymbol{B}$ ) approaches zero or if the charge is infinitely boosted (and thus $\boldsymbol{B}$ becomes orthogonal to $\boldsymbol{E})$. The first case corresponds to a static configuration, while the second one to an ultrarelativistic limit. We shall indeed find below both analogues as solutions of CS modified gravity, but we stress that the naive analogy with electrodynamics does not yet rule out other possible spinning configurations in CS modified gravity.

\section{Space of Solutions}

Before discussing some specific solutions to the CS modified field equations, let us classify the space of solutions. Figure 1 presents a 2-dimensional depiction of this space. The set $\mathcal{E}$ denotes the Einstein space of solutions, whose members have a vanishing Ricci tensor, while $\mathcal{C S}$ denotes the CS space of solutions, whose members satisfy the CS modified field equations [Eq. (16)], without necessarily being Ricci flat. The intersection of the Einstein and the CS space defines the Pontryagin space, denoted by $\mathcal{P}:=\mathcal{E} \cap \mathcal{C S}$, whose members satisfy both the Einstein and the modified field equations independently. Therefore, solutions that live in $\mathcal{P}$ possess a vanishing $\mathrm{C}$-tensor and automatically satisfy the Pontryagin constraint, while those living in $\mathcal{E} \backslash \mathcal{P}$ satisfy the vaccum Einstein equations but not the Pontryagin constraint. Moreover, solutions that live in $\mathcal{C S} \backslash \mathcal{P}$ are not Ricci-flat but do satisfy the Pontryagin constraint because they solve the modified field equations. Solutions of class $\mathcal{P}$ shall be referred to as GR solutions, while solutions of class $\mathcal{C S} \backslash \mathcal{P}$ shall be referred to as non-GR solutions. 
To date, only one non-GR solution has been found perturbatively [19] by assuming a far field expansion for point-particle sources in the PN weak-field/slow-motion approximation. We shall show in the next sections that non-GR solutions exist only in scenarios with a sufficient degree of generality, but not in highly symmetric cases. In the language of dynamical systems theory, the $\mathcal{P}$ space acts as an "attractor" of highly symmetric solutions, emptying out the $\mathcal{C S}$ space.

In view of this, let us discuss some properties of solutions that live in the $\mathcal{P}$ space. In this space, the $\mathrm{C}$-tensor simplifies to

$$
\left.C^{a b}\right|_{\mathrm{R}_{\mathrm{ab}}=0}=v_{c d}{ }^{*} R^{d(a b) c}=v_{c d}{ }^{*} C^{d(a b) c}=0,
$$

where $C_{a b c d}$ is the Weyl tensor and ${ }^{*} C$ its dual, defined in Eqs. (A2) and (A3). Equation (25) leads to three distinct possibilities:

1. The (dual) Weyl tensor vanishes. However, since class $\mathcal{P}$ members also have a vanishing Ricci tensor, this condition reduces all possible solutions to Minkowski space.

2. The covariant acceleration of $\theta$ vanishes. This condition imposes a strong restriction on the geometry (cf. e.g. [55]), which leads to spacetimes that are either flat or exhibit a null Killing vector.

3. Only the contraction of the covariant acceleration with the dual Weyl tensor vanishes.

Moreover, for solutions in $\mathcal{P}$, the vanishing of the Ricci tensor forces the Weyl tensor to be divergenceless, via the contracted Bianchi identities. These observations are a clear indication that the solutions inhabiting $\mathcal{P}$ must be special - for instance, exhibit a certain number of Killing vectors. Conversely, one may expect that solutions inhabiting $\mathcal{C S} \backslash \mathcal{P}$ cannot be "too special." We shall put these expectations on a solid basis and confirm them in the next sections.

\section{PERSISTENCE OF GR SOLUTIONS}

In this section, we study some solutions of GR that are known to persist in CS gravity [8, 22], using the insight on the Pontryagin constraint gained so far. In the language of Sec. II C we look for solutions that inhabit $\mathcal{P}$, cf. Fig. [1.

\section{A. Schwarzschild Solution}

The Schwarzschild solution,

$$
\begin{aligned}
d s^{2}=-\left(1-\frac{2 M}{r}\right) & d t^{2}+\left(1-\frac{2 M}{r}\right)^{-1} d r^{2} \\
& +r^{2}\left(d \Theta^{2}+\sin ^{2}(\Theta) d \phi^{2}\right)
\end{aligned}
$$

is also a solution of the CS modified field equations if [8]

$$
\theta=\frac{t}{\mu} \quad \rightarrow \quad v_{\mu}=[1 / \mu, 0,0,0] .
$$

We refer to Eq. (27) as the canonical choice of the CS scalar field [8]. In that case, the C-tensor can be interpreted as a 4-dimensional generalization of the ordinary 3-dimensional Cotton tensor. Moreover, spacetimedependent reparameterization of the spatial variables and time translation remain symmetries of the modified action [8].

We investigate now the most general form of $\theta=$ $\theta(t, r, \Theta, \phi)$ that will leave the Schwarzschild metric a solution of the modified theory. The Pontryagin constraint always holds, regardless of $\theta$, because the spacetime is spherically symmetric, but $C_{a b}=0$ yields non-trivial equations. Since we have chosen the Schwarzschild line element, we cannot force the (dual) Weyl tensor to vanish (option 1 in Sec. IIC), where the only linearly independent component is

$$
C_{t r \Theta \phi}=\frac{2 M}{r} \sin \Theta .
$$

Another possibility is to force the scalar field to have a vanishing covariant acceleration (option 2 in Sec. IIC). This condition then yields an over-constrained system of partial differential equations (PDEs), whose only solution for $M \neq 0$ is the trivial one: constant $\theta$. We are thus left with the remaining possibility (option 3 in Sec. (IC), namely that only the contraction of the covariant acceleration with the dual Weyl tensor vanishes. This possibility yields the following set of PDEs

$$
\theta_{, t \Theta}=\theta_{, t \phi}=\frac{\partial}{\partial r}\left(\frac{\theta_{, \Theta}}{r}\right)=\frac{\partial}{\partial r}\left(\frac{\theta_{, \phi}}{r}\right)=0,
$$

the solution of which is given by

$$
\theta=F(t, r)+r G(\Theta, \phi) .
$$

Note that this scalar field possesses a non-vanishing covariant acceleration, namely $v_{t t}, v_{t r}, v_{r r}, v_{\Theta \Theta}, v_{\Theta \phi}$ and $v_{\phi \phi}$ are non-vanishing, e.g.

$$
v_{t t}=\partial_{r r} F-\frac{M}{r^{2}}\left(1-\frac{2 M}{r}\right)\left(\partial_{r} F+G\right) .
$$

For the choice of $\theta$ given in Eq. (30) the Schwarzschild solution is always a solution of the modified theory. Note that Eq. (30) reduces to the canonical choice for $G=0$ and $F=t / \mu$, for which the only non-vanishing component of the covariant acceleration is $v_{t r}=-M /\left(r^{2} f \mu\right)$.

This simple calculation of the most general form of the scalar field that respects the Schwarzschild solution leads to two important consequences:

- The existence of specific solutions depends sensitively on the choice of the scalar field. 
- The satisfaction of the Pontryagin constraint is a necessary but not a sufficient condition for the Ctensor to vanish.

In order to illustrate the second point, let us consider the scalar field $\theta=m_{C S} \sin \Theta$, with $m_{C S}$ a constant. Then the Pontryagin constraint is still satisfied, but the C-tensor has one non-vanishing component,

$$
C_{t \phi}=\frac{3 M m_{C S}}{r^{4}} \sin ^{2} \Theta\left(1-\frac{2 M}{r}\right),
$$

and the Schwarzschild line element [Eq. (26)] is no longer a solution to the modified field equations [Eq. (16)].

\section{B. Spherically symmetric metrics}

Let us now pose the question whether there can be non-GR solutions in CS modified gravity that preserve spherical symmetry. Any line element respecting this symmetry must be diffeomorphic to (cf. e.g. [62])

$$
d s^{2}=g_{\alpha \beta}\left(x^{\gamma}\right) d x^{\alpha} d x^{\beta}+\Phi^{2}\left(x^{\gamma}\right) d \Omega_{\mathcal{S}^{2}}^{2},
$$

where $g_{\alpha \beta}\left(x^{\gamma}\right)$ is a Lorentzian 2-dimensional metric with some coordinates $x^{\gamma}, \Phi\left(x^{\gamma}\right)$ is a scalar field (often called "dilaton" or "surface radius") and $d \Omega_{\mathcal{S}^{2}}^{2}$ is a line element of the round 2-sphere, with some coordinates $x^{i}$. For such a line element, one can show straightforwardly that the Pontryagin constraint is always satisfied (cf. e.g. appendix A of [63]), and that the only non-vanishing components of the Ricci tensor are $R_{\alpha \beta}$ and $R_{i j}$. On the other hand, for the most general scalar field $\theta$, the only nonvanishing components of the C-tensor are of the form $C_{\alpha i}$. Remarkably, the C-tensor and the Ricci tensor decouple and both have to vanish independently as a consequence of the modified field equations. In other words, for spherically symmetric line elements there cannot be solutions that live in $\mathcal{C S} \backslash \mathcal{P}$. Instead all solutions are pushed to $\mathcal{P}$, which then uniquely leads to the Schwarzschild solution by virtue of the Birkhoff theorem [97].

We have just shown that for all spherically symmetric situations the vacuum solutions to the CS modified field equations live in $\mathcal{P}$, and therefore are given uniquely by the Schwarzschild solution. For non-vacuum solutions with the same symmetries, similar conclusions hold, since the field equations still decouple into non-vacuum Einstein equations and the vanishing of the $\mathrm{C}$-tensor. Therefore, all solutions are again pushed to $\mathcal{P}$ and spherically symmetric solutions of GR (such as the ReissnerNordström BH or Friedmann-Robertson-Walker spacetimes) persist in CS modified gravity, provided $\theta$ is of the form

$$
\theta=F\left(x^{\gamma}\right)+\Phi\left(x^{\gamma}\right) G\left(x^{i}\right) .
$$

This result is completely analog to Eq. (30). In all spherically symmetric scenarios, the solutions to the CS modified field equations live in $\mathcal{P}$ and the expectations of Sec. IIC hold.

\section{Losing the Kerr solution}

As an example of a relevant GR solution that does not persist in the modified theory we consider the Kerr solution. The Kerr metric yields a non-vanishing Pontryagin term [98], which in Boyer-Lindquist coordinates

$$
\begin{gathered}
d s^{2}=-\frac{\Delta-a^{2} \sin ^{2} \Theta}{\Sigma} d t^{2}-\frac{4 a M r \sin ^{2} \Theta}{\Sigma} d t d \phi \\
+\frac{\left(r^{2}+a^{2}\right)^{2}-a^{2} \Delta \sin ^{2} \Theta}{\Sigma} \sin ^{2} \Theta d \phi^{2}+\frac{\Sigma}{\Delta} d r^{2}+\Sigma d \Theta^{2}
\end{gathered}
$$

can be written as

$$
\begin{aligned}
{ }^{*} R R= & 96 \frac{a M^{2} r}{\Sigma^{6}} \cos \Theta\left(r^{2}-3 a^{2} \cos ^{2} \Theta\right) \\
& \left(3 r^{2}-a^{2} \cos ^{2} \Theta\right)
\end{aligned}
$$

with $\Sigma=r^{2}+a^{2} \cos ^{2} \Theta$ and $\Delta=r^{2}+a^{2}-2 M r$. In light of the physical interpretations of Sec. IIB one would expect this result since the Kerr spacetime possesses a complex Newman-Penrose scalar $\Psi_{2}$.

The Pontryagin constraint is satisfied in certain limits. For example, as the Kerr parameter goes to zero, $a \rightarrow$ 0 , the Schwarzschild solution is recovered and the righthand side of Eq. (36) vanishes. Similarly, in the limit as the mass goes to zero, $M \rightarrow 0$, the right-hand side of Eq. (36) also vanishes. However, for any finite $a$ and $M$ the Pontryagin term is non-vanishing and, thus, the Kerr spacetime cannot be a solution to the CS modified field equations 33.

What line element then replaces the Kerr solution in the modified theory? A reasonable attempt to construct a spinning $\mathrm{BH}$ in $\mathrm{CS}$ gravity is to consider generic axisymmetric and either static or stationary line elements, which we shall investigate in the next sections.

\section{STATIC, AXISYMMETRIC SOLUTIONS}

Before embarking on a tour de force through generic stationary and axisymmetric solutions, we shall first consider the simpler case of static and axisymmetric solutions. Following [64], the most general static and axisymmetric line element is diffeomorphic to

$$
d s^{2}=-V d t^{2}+V^{-1} \rho^{2} d \phi^{2}+\Omega^{2}\left(d \rho^{2}+\Lambda d z^{2}\right),
$$

where we have three undetermined functions of two coordinates: $V(\rho, z), \Omega(\rho, z)$ and $\Lambda(\rho, z)$. The two commuting Killing fields, $\xi^{a}=\left(\partial_{t}\right)^{a}$ and $\psi^{a}=\left(\partial_{\phi}\right)^{a}$, are associated with stationarity and axisymmetry respectively. However, since there is no cross-term $d t d \phi$, the line element of Eq. (37) is not just stationary but also static. The 
components of its Ricci tensor are given by

$$
\begin{aligned}
& R_{t \phi}=R_{t \rho}=R_{t z}=R_{\phi \rho}=R_{\phi z}=0 \text {, } \\
& R_{t t}=\frac{1}{2 \Omega^{2}}\left[V_{, \rho \rho}+\frac{V_{, z z}}{\Lambda}+\frac{V_{, \rho}}{\rho}-\frac{V_{, \rho}^{2}}{V}-\frac{V_{, z}^{2}}{V \Lambda}\right. \\
& \left.+\frac{V_{, \rho} \Lambda_{, \rho}}{2 \Lambda}-\frac{V_{, z} \Lambda_{, z}}{2 \Lambda^{2}}\right] \\
& R_{\phi \phi}=\frac{1}{2 \Omega^{2}}\left[\frac{\rho^{2} V_{, \rho \rho}}{V^{2}}+\frac{\rho^{2} V_{, z z}}{V^{2} \Lambda}+\frac{\rho V_{, \rho}}{V^{2}}-\frac{\rho^{2} V_{, \rho}^{2}}{V^{3}}-\frac{\rho^{2} V_{, z}^{2}}{V^{3} \Lambda}\right. \\
& \left.-\frac{\rho \Lambda_{, \rho}}{V \Lambda}+\frac{\rho^{2} \Lambda_{, \rho} V_{, \rho}}{2 V^{2} \Lambda}-\frac{\rho^{2} \Lambda_{, z} V_{, z}}{2 V^{2}}\right], \\
& R_{\rho \rho}=\frac{V_{, \rho}}{\rho V}-\frac{V_{, \rho}^{2}}{2 V^{2}}-\frac{\Omega_{, \rho \rho}}{\Omega}-\frac{\Omega_{, z z}}{\Lambda \Omega}+\frac{\Omega_{, \rho}}{\rho \Omega}+\frac{\Omega_{, \rho}^{2}}{\Omega^{2}}+\frac{\Omega_{, z}^{2}}{\Lambda \Omega^{2}} \\
& -\frac{\Lambda_{, \rho \rho}}{2 \Lambda}+\frac{\Lambda_{, \rho}^{2}}{4 \Lambda^{2}}-\frac{\Omega_{, \rho} \Lambda_{, \rho}}{2 \Lambda \Omega}+\frac{\Omega_{, z} \Lambda_{, z}}{2 \Lambda^{2} \Omega}, \\
& R_{z z}=-\frac{V_{, z}^{2}}{2 V^{2}}-\frac{\Lambda \Omega_{, \rho \rho}}{\Omega}-\frac{\Omega_{, z z}}{\Omega}-\frac{\Omega_{, \rho} \Lambda}{\rho \Omega}+\frac{\Omega_{, \rho}^{2} \Lambda}{\Omega^{2}}+\frac{\Omega_{, z}^{2}}{\Omega^{2}} \\
& -\frac{\Lambda_{, \rho \rho}}{2}-\frac{\Lambda_{, \rho}}{2 \rho}+\frac{\Lambda_{, \rho}^{2}}{4 \Lambda}-\frac{\Omega_{, \rho} \Lambda_{, \rho}}{2 \Omega}+\frac{\Omega_{, z} \Lambda_{, z}}{2 \Omega \Lambda}, \\
& R_{\rho z}=-\frac{V_{, z} V_{, \rho}}{2 V^{2}}+\frac{V_{, z}}{2 \rho V}+\frac{\Omega_{, z}}{\rho \Omega},
\end{aligned}
$$

and exhibit only five non-vanishing components. With the canonical choice of the CS scalar field [Eq. (27)] it is now straightforward to check that the five corresponding components of the C-tensor vanish,

$$
C_{t t}=C_{\phi \phi}=C_{\rho \rho}=C_{z z}=C_{\rho z}=0 .
$$

As in the spherically symmetric case, we are faced with the remarkable consequence that the field equations [Eq. (16)] decouple into the vacuum Einstein equations plus the vanishing of the $\mathrm{C}$-tensor, viz.

$$
R_{a b}=0, \quad C_{a b}=0 .
$$

In other words, using the classification of Sec. IIC, all static and axisymmetric solutions live in $\mathcal{P}$, which again confirms previous expectations.

With these considerations in mind, we can now simplify the line element of Eq. (37). From [64], the function $\Lambda$ can be chosen to be constant, e.g. $\Lambda=1$, and therefore the line-element reduces to the Weyl class,

$$
d s^{2}=-e^{2 U} d t^{2}+e^{-2 U}\left[e^{2 k}\left(d \rho^{2}+d z^{2}\right)+\rho^{2} d \phi^{2}\right] .
$$

The vacuum Einstein equations then simplify to

$$
\Delta U=0, \quad k_{, \rho}=\rho\left(U_{, \rho}^{2}-U_{, z}^{2}\right), k_{, z}=2 \rho U_{, \rho} U_{, z},
$$

where $\Delta=\partial^{2} / \partial \rho^{2}+1 / \rho \partial / \partial_{\rho}+\partial^{2} / \partial z^{2}$ is the flat space Laplacian in cylindrical coordinates. The function $U$ thus solves a Laplace equation, and for any such solution the function $k$ can be determined by a line integral [55].

The Pontryagin constraint is fulfilled automatically for all line elements diffeomorphic to Eq. (46), but as we have seen in the previous sections, this is not sufficient to achieve $C_{a b}=0$. For example, with the choices [65, 66] ( $m$ is constant)

$$
U=-\frac{m}{\sqrt{\rho^{2}+z^{2}}}, \quad k=-\frac{m^{2} \rho^{2}}{2\left(\rho^{2}+z^{2}\right)^{2}},
$$

the vacuum Einstein equations hold and the Pontryagin constraint is fulfilled, but the C-tensor has the nonvanishing components

$$
C_{\rho \phi}=\frac{2 m^{4} \rho^{3} z}{\mu\left(\rho^{2}+z^{2}\right)^{5}} \exp \left[\frac{m^{2} \rho^{2}}{\left(\rho^{2}+z^{2}\right)^{2}}\right], \quad C_{z \phi}=\frac{1}{2 \rho z} C_{\rho \phi} .
$$

Since the C-tensor must vanish independently from the Einstein equations, once more we are faced with three distinct possibilities, identical to those described in Sec. II C. The first possibility (option 1 in Sec. IIC) is to demand that the Weyl tensor vanishes, but since also the Ricci tensor vanishes, the spacetime would have to be flat. The second possibility (option 2 in Sec. II C) is to demand that the covariant acceleration of $\theta$ vanishes, i.e., $\nabla_{a} \theta$ is a covariantly constant vector. However, as we have mentioned already, a vacuum solution with a covariantly constant vector field must be either flat, or the vector must be a null-vector. The first alternative is trivial, while the second one is not particularly interesting in the context of static axisymmetric spacetimes. We shall discuss the latter possibility further in Sec. VIA

The only remaining possibility (option 3 in Sec. IIC) is for the contraction of the covariant acceleration and the dual Weyl tensor to vanish. The C-tensor can then be simplified to

$$
C^{a b} \propto \Gamma_{\rho t}^{t}{ }^{*} C^{t(a b) \rho}+\Gamma_{z t}^{t}{ }^{*} C^{t(a b) z}=0,
$$

which has only two non-vanishing components. Using the Einstein equations to simplify these expressions we obtain a set of nonlinear PDEs,

$$
\begin{aligned}
& U_{, \rho z} U_{, \rho}+U_{, z z} U_{, z}=2 \rho\left(U_{, z}^{3} U_{, \rho}+U_{, \rho}^{3} U_{, z}\right)-\frac{2}{\rho} U_{, \rho} U_{, z}, \\
& U_{, \rho z} U_{, z}-U_{, z z} U_{, \rho}=\rho\left(U_{, \rho}^{4}-U_{, z}^{4}\right)-\frac{1}{\rho} U_{, \rho}^{2} .
\end{aligned}
$$

We used Maple to obtain some solutions to these PDEs. The Schwarzschild solution

$$
U=\frac{1}{2} \ln \frac{\sqrt{\rho^{2}+(z+M)^{2}}+\sqrt{\rho^{2}+(z-M)^{2}}-2 M}{\sqrt{\rho^{2}+(z+M)^{2}}+\sqrt{\rho^{2}+(z-M)^{2}}+2 M}
$$

of course solves these PDEs. Some other simple solutions are $U=U_{0}, U=U_{0} \pm \ln \rho$ and $U=U_{0}+$ $\ln \left(\sqrt{\rho^{2}+z^{2}}+z\right) / 2$, where $U_{0}$ is a constant. Not only do these solution yield a vanishing Ricci tensor, but they also yield a vanishing Riemann tensor, which shows they are Minkowski spacetime in disguise. In addition to these trivial solutions, there exist exactly two more:

$$
d s^{2}=-\frac{1}{z} d t^{2}+z d z^{2}+z^{2}\left(d \rho^{2}+\rho^{2} d \phi^{2}\right)
$$


and

$$
\begin{aligned}
d s^{2} & =-\left(\frac{2 m}{z}-1\right) d t^{2}+\left(\frac{2 m}{z}-1\right)^{-1} d z^{2} \\
& +z^{2}\left(d \rho^{2}+\sinh ^{2} \rho d \phi^{2}\right)
\end{aligned}
$$

While these solutions certainly are non-trivial, neither the first [Eq. (53)] nor the second [Eq. (54)] solution is physically relevant. The former has a naked singularity at $z=0$, while the latter, whose singularity at $z=0$ is screened by a Killing horizon at $z=2 m=$ const., possesses a Killing vector $k^{a}=\left(\partial_{t}\right)^{a}$ that is spacelike in the "outside" region $z>2 m$, i.e. $g_{a b} k^{a} k^{b}=1-2 m / z>0$.

Let us now prove that these are the only solutions to the modified field equations. The crucial observation is that the spatial part of the C-tensor for static, axisymmetric spacetimes reduces to the 3-dimensional Cotton tensor [8]. This tensor vanishes if and only if the corresponding 3-dimensional space (spatial sector of the 4dimensional metric) is conformally flat, i.e. $g_{i j}=\Phi \delta_{i j}$, where the conformal factor $\Phi$ is a function of the coordinates and $\delta_{i j}$ is the spatial part of the Minkowski metric. We may then exploit a result by Lukács and Perjés [67] that the line elements of Eqs. (53), (54) and (26) are the only static and axisymmetric solutions that are spatially conformally flat. Therefore, it follows that these equations are the only solutions to the modified field equations.

The above considerations also apply to more general CS scalar fields. All simplifications hinge on the decoupling of the modified field equations, which occurs if and only if $\theta_{, t \phi}=0$. We can solve this PDE to obtain

$$
\theta=\theta_{1}(t, \rho, z)+\theta_{2}(\rho, z, \phi) .
$$

For all scalar fields of this form, the modified field equations decouple and the C-tensor has five non-vanishing components, which define a system of PDEs for one of the two arbitrary functions $k$ or $U$. However, we do not expect more solutions to arise in this way, since this case leads to the same constraints as the canonical one, plus three extra PDEs, which essentially compensate the freedom to tinker with the two arbitrary functions in Eq. (55).

The most general CS scalar field, however, does not allow for a decoupling of the type described above. If the scalar field has $\theta_{t \phi} \neq 0$, then the $(\rho, \rho),(\rho, z)$ and $(z, z)$ components of the modified field equations do not decouple. However, the $(t, t)$ and $(\phi, \phi)$ components still do decouple because the corresponding C-tensor components vanish. The equation

$$
R_{t}^{t}+R_{\phi}^{\phi}=-\frac{1}{2} \frac{\Lambda_{, \rho}}{\Lambda \rho^{2} \Omega^{2}}=0,
$$

forces $\Lambda$ to be a function of $z$ alone. Through a diffeomorphism, this function can be set to unity, as argued in 64.

The modified field equations are too difficult to solve analytically with Maple, so in order to study solutions that do not lead to a decoupling of the modified field equations, we shall assume for simplicity $\theta=\tilde{\theta}(t, \phi)$. From the Ricci sector of the field equations $\left(R_{t t}=0=\right.$ $\left.R_{\phi \phi}\right)$ we find that $U$ is again a solution of $\Delta U=0$. We can use this relation to simplify the C-tensor, and the ensuing equations $C_{t \phi}=C_{t \rho}=C_{t z}=C_{\phi \rho}=C_{\phi z}=0$ lead to a system of second order PDEs for $\theta$ and $k$. We investigated this system with Maple and found that solutions exist if and only if $\theta$ is a function of only one variable, i.e. $\theta=\theta(t)$ or $\theta=\theta(\phi)$. These results indicate that there are no solutions of the modified field equations if $\theta$ is bivariate.

In summary, we have shown in this section that the field equations decouple if the CS scalar field solves $\theta_{, t \phi}=$ 0 , and their solution is the Schwarzschild $\mathrm{BH}$ and two additional (unphysical) solutions [Eqs. (53) and (54)]. For CS fields that satisfy $\theta_{, t \phi} \neq 0$, the modified field equations do not seem to have a solution. Therefore, there are no static and axisymmetric solutions in CS gravity, apart from the Schwarzschild BH and some unphysical solutions, irrespective of the CS scalar field.

\section{STATIONARY, AXISYMMETRIC SOLUTIONS}

\section{A. General line elements}

Equipped with the tools from the previous section, we drop the requirement of staticity and replace it by the weaker one of stationarity. In essence, this means that we shall allow the gravitomagnetic sector of the metric to be different from zero. The most general, stationary and axisymmetric line-element is diffeomorphic to [55]

$d s^{2}=-V(d t-w d \phi)^{2}+V^{-1} \rho^{2} d \phi^{2}+\Omega^{2}\left(d \rho^{2}+\Lambda d z^{2}\right)$,

where the functions $V, w, \Omega$ and $\Lambda$ depend on $\rho$ and $z$, only. This line element is identical to Eq. (37) as $w \rightarrow 0$. In GR, the function $w$ can be identified with the angular velocity. The Ricci tensor for this line element is similar 
to $R_{a b}^{\text {static }}$ [Eqs. (38)-(43)] and its components are

$$
\begin{aligned}
R_{t \rho} & =R_{t z}=R_{\phi \rho}=R_{\phi z}=0 \\
R_{t t} & =R_{t t}^{\text {static }}+\left(w_{, \rho}^{2}+\frac{w_{, z}^{2}}{\Lambda}\right) \frac{V^{3}}{2 \rho^{2} \Omega^{2}}, \\
R_{t \phi} & =\frac{w}{2 \Omega^{2}}\left[-V_{, \rho \rho}-\frac{V_{, z z}}{\Lambda}-\frac{V_{, \rho}}{\rho}+\frac{V_{, \rho}^{2}}{V}+\frac{V_{, z}^{2}}{V \Lambda}\right. \\
& -2 \frac{V_{, \rho} w_{, \rho}}{w}-2 \frac{V_{, z} w, z}{w \Lambda}-\frac{w_{, \rho \rho} V}{w}-\frac{w_{, z z} V}{w \Lambda} \\
& +\frac{w_{, \rho} V}{\rho w}-\frac{w_{, \rho}^{2} V^{3}}{\rho^{2}}-\frac{w_{, z}^{2} V^{3}}{\rho^{2} \Lambda} \\
& \left.-\frac{\Lambda_{, \rho} V_{, \rho}}{2 \Lambda}+\frac{\Lambda_{, z} V_{, z}}{2 \Lambda^{2}}-\frac{\Lambda_{, \rho} w_{, \rho} V}{2 w \Lambda}+\frac{\Lambda_{, z} w_{, z} V}{2 w \Lambda^{2}}\right], \\
R_{\rho \rho} & =R_{\rho \rho}^{\text {static }}+w_{, \rho}^{2} \frac{V^{2}}{2 \rho^{2}}, \\
R_{z z} & =R_{z z}^{\text {static }}+w_{, z}^{2} \frac{V^{2}}{2 \rho^{2}}, \\
R_{\rho z} & =R_{\rho z}^{\text {static }}+w_{, \rho} w_{, z} \frac{V^{2}}{2 \rho^{2}}, \\
R & =R^{\text {static }}+\left(w_{, \rho}^{2}+\frac{1}{\Lambda} w_{, z}^{2}\right) \frac{V^{2}}{2 \rho^{2} \Omega^{2}} .
\end{aligned}
$$

The somewhat lengthy component $R_{\phi \phi}$ can be deduced from $R$ and the other components. The quantity $R^{\text {static, }}$

$$
\begin{aligned}
R^{\text {static }}= & \frac{1}{2 \Omega^{2}}\left[2 \frac{V_{\rho}}{V \Lambda}-\frac{V_{\rho}^{2}}{V^{2}}-\frac{V_{z}^{2}}{V^{2} \Lambda}\right. \\
& -4 \frac{\Omega_{\rho \rho}}{\Omega}-4 \frac{\Omega_{z z}}{\Lambda \Omega}+4 \frac{\Omega_{\rho}^{2}}{\Omega^{2}}+4 \frac{\Omega_{z}^{2}}{\Lambda \Omega^{2}} \\
- & \left.2 \frac{\Lambda_{\rho \rho}}{\Lambda}-2 \frac{\Lambda_{\rho}}{\rho \Lambda}+\frac{\Lambda_{\rho}^{2}}{\Lambda^{2}}-2 \frac{\Lambda_{\rho} \Omega_{\rho}}{\Lambda \Omega}+2 \frac{\Lambda_{z} \Omega_{z}}{\Lambda^{2} \Omega}\right],
\end{aligned}
$$

is the trace of Eqs. (38)-(43).

As before, let us begin with the canonical choice for the CS scalar field, namely Eq. (27). Then the only nonzero components of the C-tensor are $C_{\rho t}, C_{z t}, C_{\rho \phi}$ and $C_{z \phi}$. As in the previous cases, there is a decoupling of the field equations that allows us to set $\Lambda=1$ and to consider the slightly simpler line element (Lewis-Papapetrou-Weyl metric)

$d s^{2}=-e^{2 U}(d t-w d \phi)^{2}+e^{-2 U}\left[e^{2 k}\left(d \rho^{2}+d z^{2}\right)+\rho^{2} d \phi^{2}\right]$,

where again the functions $U, k$ and $w$ depend on the coordinates $\rho$ and $z$ only. With this line element, the last lines vanish in the multi-line expressions for the Ricci tensor, Eqs. (39)-(42), (60) and (65), because $\Lambda=1$. The vacuum Einstein equations simplify considerably with $\Lambda=1$. Essentially, they are similar to Eq. (47) but with a complicated source and an additional equation for $w$. Even within GR, explicit solution to this set of PDEs can only be found in certain special cases [55].

The Pontryagin constraint for the line element of Eq. (66) is not satisfied in general. This constraint yields a complicated second order PDE for $w, U$ and $k$, presented in appendix $B$, which of course is trivially satisfied as $w \rightarrow 0$. Certain solutions to the PDE in appendix $\mathrm{B}$ can be obtained, e.g. $\left(\bar{w}:=e^{2 U} w\right)$

$$
\begin{array}{lll}
k=k(\rho, z) & \bar{w}=c_{1} e^{2 U}, & U=U(\rho, z), \\
k=k(\rho, z) & \bar{w}= \pm \rho, & U=U(\rho, z), \\
k=\ln (\rho)+\tilde{k}(z), & \bar{w}=\tilde{w}(z) \rho, & U=\frac{1}{2} \ln (\rho)+c_{1}, \\
k=k(\rho), & \bar{w}=\bar{w}(\rho), & U=U(\rho),
\end{array}
$$

where $c_{1}$ is a constant. The first line reduces to static solutions upon redefining $t^{\prime}=t-c_{1} \phi$. The second line leads to metrics of Petrov type $I I$, the so-called van Stockum class, which we shall discuss in Sec.VB. The third line of Eq. (67) cannot be made to solve the modified field equations. The last line implies cylindrical symmetry, which again via the field equations leads to flat spacetime. We have thus been unable to find non-trivial solutions either by hand or using symbolic manipulation software [99].

Unlike the previous section, we cannot provide here a truly exhaustive discussion of all solutions of the decoupled field equations. This is because $C_{a b}=0$ does not necessarily imply spatial conformal flatness for the stationary case. Based on the evidence found so far, it seems unlikely that there are other non-trivial and physically interesting solutions besides the static ones. This is because the vacuum Einstein equations $\left[R_{a b}=0\right]$ already determine the function $k$ uniquely up to an integration constant, and also impose strong restrictions on the functions $U$ and $w[55]$. The constraints $C_{a b}=0 \mathrm{im}-$ pose four additional conditions on these functions that can be found in [68]. Since the system of partial differential equations is over-constrained, it is unlikely that additional solutions exist. Therefore, whenever the field equations decouple into $R_{a b}=0=C_{a b}$ we do not expect physically relevant solutions besides the Schwarzschild one and its flat space limit.

The decoupling exhibited by the modified field equations does not occur only for the canonical choice of the CS scalar field. In order for such a decoupling to occur, the following system of PDEs must be satisfied:

$$
\theta_{, t t}=\theta_{, \phi \phi}=\theta_{, t \phi}=\theta_{, \rho}=\theta_{, z}=0,
$$

which yields the solution

$$
\theta=\frac{t}{\mu}+\frac{\phi}{\nu}
$$

with constant $\mu, \nu$. The canonical choice is recovered as $\nu \rightarrow \infty$.

But what if the scalar field is not of the form of Eq. (69)? In this case, the field equations do not decouple and solving the entire system is much more complicated. However, we can deduce from Eq. (58) that still the four C-tensor components $C_{\rho t}, C_{z t}, C_{\rho \phi}$ and $C_{z \phi}$ have to vanish. Therefore, even though no decoupling occurs, the same issue of an over-constrained system of equations does arise, analogous to the one in Sec. $\mathrm{VA}$. Even with 
this generalization, it is still quite difficult to find solutions to the coupled system. In general, one might be able to find solutions both of class $\mathcal{P}$ and class $\mathcal{C S} \backslash \mathcal{P}$ because non-canonical CS fields allow for general $\theta$, which entails a new degree of freedom. We shall see in Sec. VB that for a simplified subclass of stationary and axisymmetric line elements, which automatically satisfy the Pontryagin constraint, solutions can indeed be found, including mathematical BHs.

\section{B. Van Stockum line element}

We study now a slightly less general line element that still is stationary and axisymmetric, namely the van Stockum line element [55]

$$
d s^{2}=\rho \Omega d t^{2}-2 \rho d t d \phi+\frac{1}{\sqrt{\rho}}\left(d \rho^{2}+d z^{2}\right),
$$

where the only arbitrary function is $\Omega=\Omega(\rho, z)$. The metric is different from that considered in Eq. (66) since it does not possess a $d \phi^{2}$ component. The only nonvanishing component of the Ricci tensor for such a spacetime is

$$
R_{t t}=-\frac{\rho^{3 / 2}}{2} \Delta \Omega
$$

where again $\Delta$ is the flat space Laplacian in cylindrical coordinates.

The Pontryagin constraint is automatically satisfied for the van Stockum line element even though it is of Petrov type $I I$, precisely because of the vanishing $d \phi^{2}$ term. The $t t$ component of the modified equations then determines $\Omega$, and this forces all other components of the C-tensor to vanish, except for $C_{t \phi}$ and $C_{\phi \phi}$ that are automatically zero. These constraints act as a system of PDEs for the scalar field, whose unique solution is $\theta=\theta(\rho, z)$. Note that the canonical choice for $\theta$ is not compatible with the van Stockum line element. The remaining PDE $R_{t t}+C_{t t}=0$ can be solved for $\theta$ and $\Omega$, where $C_{t t}$ now simplifies to

$$
\begin{aligned}
C_{t t} & =\frac{\rho^{2}}{2}\left[\left(\theta_{, \rho \rho}-\theta_{, z z}\right)\left(\Omega_{, \rho z}+\frac{3}{4 \rho} \Omega_{, z}\right)\right. \\
& +\theta_{, \rho}\left(\Omega_{, z z z}+\Omega_{, \rho \rho z}+\frac{3}{2 \rho} \Omega_{, \rho z}+\frac{3}{8 \rho^{2}} \Omega_{, z}\right) \\
& -\theta_{, z}\left(\Omega_{, \rho \rho \rho}+\Omega_{, \rho z z}+\frac{9}{4 \rho} \Omega_{, \rho \rho}+\frac{3}{4 \rho} \Omega_{, z z}+\frac{3}{8 \rho^{2}} \Omega_{, \rho}\right) \\
& \left.-\theta_{, \rho z}\left(\Omega_{, \rho \rho}-\Omega_{, z z}+\frac{3}{2 \rho} \Omega_{, \rho}\right)\right] .
\end{aligned}
$$

Combining this with $R_{t t}$ from Eq. (71) we find two simple solutions of Eq. (16):

$$
\Omega=c, \quad \theta=\theta(\rho, z),
$$

where $c$ is a constant and

$$
\Omega=c+\frac{d}{\sqrt{\rho}}, \quad \theta=\frac{2}{3} \sqrt{\rho} z+\tilde{\theta}(\rho),
$$

where $c$ and $d$ are constants [100]. Equation (73) leads to zero Ricci and C-tensor separately and it is thus a GR solution that belongs to the subspace $\mathcal{P}$. The ensuing metric is exceptional in that it has a third Killing vector, $t \partial_{t}-\phi \partial_{\phi}+c t \partial_{\phi}$. Some of the non-vanishing Riemann tensor components for this geometry are

$$
R_{t \rho t \rho}=\frac{c}{8 \rho}, \quad R_{t \rho \rho \phi}=\frac{1}{8 \rho}, \quad R_{t \phi t \phi}=\frac{1}{4} \sqrt{\rho} .
$$

On the other hand, Eq. (74) is perhaps even more interesting since it is not Ricci-flat, but has one non-vanishing component of the Ricci tensor,

$$
R_{t t}=-\frac{d}{8 \rho}=-C_{t t} .
$$

This solution is thus a non-GR solution and it belongs to the subspace $\mathcal{C S} \backslash \mathcal{P}$. Some of the non-vanishing components of the Riemann tensor for this solution are

$$
R_{t \rho t \rho}=\frac{d+2 c \sqrt{\rho}}{16 \rho^{3 / 2}}, \quad R_{t \rho \rho \phi}=\frac{1}{8 \rho}, \quad R_{t \phi t \phi}=\frac{1}{4} \sqrt{\rho} .
$$

Notice that such a solution can represent a mathematical $\mathrm{BH}$, provided $\Omega$ vanishes for some $\rho$, i.e. a Killing horizon emerges. We call these configurations "mathematical BHs" because they are physically not very relevant: the Killing vector generating axial symmetry is light-like, as evident from (70), and the spacetime admits closed timelike curves which are not screened by a horizon [55]. For $c=1$ and $d=-2 m$ we recover (2).

Let us then summarize the most important conclusions of this section. We have investigated stationary and axisymmetric solutions to the modified field equations. We found that, for the canonical choice of $\theta$, it is unlikely that solutions can be found that differ from Minkowski and Schwarzschild. Nonetheless, for non-canonical choices of this scalar, solutions must exist. This conclusion derives from the investigation of a slightly less general stationary and axisymmetric metric, namely that of van Stockum. For this line element we found a solution to the modified field equations that lives in $\mathcal{P}$ and a family of solutions that live in $\mathcal{C S} \backslash \mathcal{P}$, both with non-canonical CS scalar fields. To our knowledge, this is the first time an exact non-GR solution is found for CS modified gravity, which in particular can represent mathematical BH configurations.

\section{BEYOND THE CANON}

We have failed in finding an exact, stationary and axisymmetric solution to the CS modified field equations representing a physical spinning $\mathrm{BH}$. A solution, however, already exists for a similar line element, albeit in a perturbative sense. In [19] and later in [21], a far-field solution to the CS modified field equations with a canonical CS scalar field was found in the weak-field/slow-motion approximation. This solution is identical to the far-field 
expansion of the Kerr solution, except for the addition of two new components in the gravitomagnetic sector of the metric $g_{0 i}$. These components vanish in GR, since only one component is required and it is aligned with the angular momentum of the spinning source. In CS gravity, the remaining components of $g_{0 i}$ are proportional to the curl of the spin angular momentum, thus breaking axisymmetry but preserving stationarity. Such a stationary, but non-axisymmetric BH will not emit gravitational waves, but it will possess a non-trivial multipolar structure, with probably more than just two nonvanishing multipoles. Such a far-field structure suggests that perhaps the only way to obtain an analog to the Kerr solution in CS gravity is to relax either the assumption of axisymmetry or stationarity. Alternatively, the van Stockum example suggests that another possibility is to allow for a general CS scalar field. In this case, however, the line element must significantly differ from the Kerr metric such that it satisfies the Pontryagin constraint [101]. We shall explore these possibilities in this section.

\section{A. Killing embedding}

We study now the possibility that the 'embedding coordinate', i.e., the velocity of the CS scalar field $\theta$, is a Killing vector. Then, $v_{a}$ is covariantly conserved because of the Killing equation $\left(\nabla_{(a} v_{b)}=0\right)$ and the fact that the connection is torsion-free $\left(\nabla_{[a} v_{b]}=\nabla_{[a} \nabla_{b]} \theta=0\right)$. This puts a strong restriction on spacetime, which for a time-like $v_{a}$ yields line elements that are diffeomorphic to

$$
d s^{2}=-d t^{2}+g_{i j}\left(x^{k}\right) d x^{i} d x^{j},
$$

where $i$ and $j$ range over all coordinates except time. Actually, Eq. (78) describes a special class of static spacetimes. When studying static solutions to the CS modified field equations with timelike $v_{a}$ in Sec. IV] we found no physically relevant solution besides Schwarzschild. The same conclusions hold here, except that we do not even recover Schwarzschild, so this route is not a promising one. A similar discussion applies to spacelike Killing vectors.

A more interesting situation arises if the vector field $v^{a}$ is a null Killing vector, $v^{a} v_{a}=\nabla_{(a} v_{b)}=0$. In this case, we get in an adapted coordinate system the line element

$$
d s^{2}=-2 d v d x^{1}+g_{i j}\left(x^{k}\right) d x^{i} d x^{j}
$$

Once again, the Pontryagin constraint is immediately satisfied, the Ricci tensor has non-vanishing $R_{i j}$ components, but no components of the C-tensor vanish. Even when we pick a simple null Killing embedding, e.g. $v_{a}=(0, \chi, 0,0)$ with $\chi=$ const., the $\mathrm{C}$-tensor has complicated spatial non-vanishing components and the modified field equations are too difficult to solve in full generality. Therefore, we focus instead on an interesting special case in the next subsection.

\section{B. pp-waves and boosted black holes}

As suggested at the end of Sec. IIB it might be possible to find solutions to the modified field equations if one considers line elements that represent exact gravitational wave solutions (pp-waves [69]). The line element for these waves is

$$
d s^{2}=-2 d v d u-H(u, x, y) d u^{2}+d x^{2}+d y^{2},
$$

which is simply a special case of the line elements considered in the previous subsection [Eq. (79)]. Particular examples of physical scenarios that are well-represented by Eq. (80) are the Aichelburg-Sexl limits [70] of various BHs. In essence, this limit is an ultrarelativistic boost that keeps the energy of the $\mathrm{BH}$ finite by taking a limit where its mass vanishes while the boost velocity approaches the speed of light. In particular, Eq. (80) can be used to represent ultrarelativistic boosts of the Kerr BH [71, 72].

Is it conceivable that a Kerr $\mathrm{BH}$ that moves ultrarelativistically solves the modified field equations, even though the Kerr BH does not? One of the main problems with the Kerr metric is that it does not satisfy the Pontryagin constraint, cf. Eq. (36), but that constraint is trivially satisfied as $M \rightarrow 0$. Nonetheless, the satisfaction of the Pontryagin constraint is only a necessary condition, but not a sufficient one, to guarantee that the modified field equations are also satisfied.

In order to study this issue, let us find the appropriate expressions for the Ricci and C-tensors. The only non-vanishing component of the Ricci tensor for the line element of Eq. (80) is given by

$$
R_{u u}=\Delta H, \quad \Delta:=\frac{\partial^{2}}{\partial x^{2}}+\frac{\partial^{2}}{\partial y^{2}} .
$$

In general, the components $C_{u x}, C_{u y}, C_{x x}, C_{y y}, C_{x y}$ are all non-vanishing and form a system of PDEs for $H$ and $\theta$. The $C_{x x}, C_{y y}$ and $C_{x y}$ components are given by

$$
C_{y y}=-C_{x x}=\theta_{, v v} H_{, x y}, \quad C_{x y}=\frac{1}{2} \theta_{, v v}\left(H_{, x x}-H_{, y y}\right) .
$$

Let us first look for GR-solutions of class $\mathcal{P}$, such that $R_{a b}=0$ and $C_{a b}=0$ independently. Since $C_{a b}=0$, there are two possibilities here: either $\theta_{, v v}=0$ or $H_{, x y}=0=$ $H_{, x x}-H_{, y y}$. In the latter case, $H$ is constrained to

$$
H=\frac{1}{2}\left(x^{2}+y^{2}\right) A(u)+x B(u)+y C(u)+D(u),
$$

which also forces $C_{u x}$ and $C_{u y}$ to vanish. The only component of the field equations left is $(u, u)$, which upon simplification with Eq. (83) yields $C_{u u}=0$ and $R_{u u}=2 A(u)$, so that $A(u)=0$. We have then found the solution

$$
H=x B(u)+y C(u)+D(u), \quad \theta=\theta(u, v, x, y),
$$


to the modified field equations. However, this solution is nothing but flat space in disguise.

Another possibility to find GR-solutions is to pick $\theta$ such that $C_{x x}, C_{y y}$ and $C_{x y}$ vanish, i.e. $\theta_{, v v}=0$. This condition leads to

$$
\theta=\lambda(u, x, y) v+\tilde{\theta}(u, x, y)
$$

The remaining non- $(u, u)$ components of the C-tensor lead to

$$
\begin{aligned}
& C_{u x}=0 \quad \rightarrow \quad \lambda_{, x} H_{, x y}=\lambda_{, y} H_{, y y} \\
& C_{u y}=0 \quad \rightarrow \quad \lambda_{, x} H_{, y y}=\lambda_{, y} H_{, x x}
\end{aligned}
$$

where we have used $R_{u u}=0$. The solution to this system of PDEs leads either to flat spacetime or to

$$
\lambda(u, x, y)=\lambda(u)
$$

Choosing Eq. (88), the remaining modified field equation [the $(u, u)$ component] becomes

$$
\begin{aligned}
\Delta H & =0, \\
2 H_{, y y} \tilde{\theta}_{, x y} & =H_{, x y}\left(\tilde{\theta}_{, y y}-\tilde{\theta}_{, x x}\right) .
\end{aligned}
$$

For some $H$ that solves the Einstein equations [i.e. the Laplace equation in Eq. [89]), the C-tensor yields a PDE for $\tilde{\theta}[\mathrm{Eq}$. (90)]. Thus we conclude that we can lift any pp-wave solution of the vacuum Einstein equations to a pp-wave solution of CS modified gravity (of class $\mathcal{P}$ ) by choosing $\theta$ such that Eqs. (85), (88)-(90) hold.

Let us give an example of this method to generate CS solutions by studying ultrarelativistically boosted Kerr BHs, for which

$$
H=h_{0} \delta(u) \ln \left(x^{2}+y^{2}\right)
$$

satisfies Eq. (89). In Eq. (91), $\delta(u)$ is the Dirac delta function and $h_{0}$ is a constant. Inserting this $H$ into Eq. (90) we find

$$
\tilde{\theta}=x \alpha\left(\frac{y}{x}\right)+\beta\left(x^{2}+y^{2}\right)
$$

where $\alpha$ and $\beta$ are arbitrary functions of their respective arguments $(y / x)$ and $\left(x^{2}+y^{2}\right)$. Equation (92), together with Eq. (85) and (88), give the full solution for the CS scalar field. We have therefore lifted the boosted Kerr BH to a solution of the modified field equations of class $\mathcal{P}$ by choosing the CS scalar field appropriately. For $\theta=\lambda v$ we recover Eq. (3).

Let us now search for non-GR solutions to the modified field equations. Since all equations decouple except for the $(u, u)$ component, we must enforce that the non $(u, u)$-components of the $\mathrm{C}$-tensor vanish, i.e. $\theta_{, v}=0$, which leads to

$$
\theta=\tilde{\theta}(u, x, y)
$$

With Eq. (93), the only component of the modified field equations left is again the $(u, u)$ one, which simplifies to a linear third order PDE:

$$
\begin{aligned}
& \left(1+\tilde{\theta}_{, y} \partial_{, x}-\tilde{\theta}_{, x} \partial_{, y}\right) \Delta H= \\
& \quad=\left(\tilde{\theta}_{, x x}-\tilde{\theta}_{, y y}\right) H_{, x y}-\left(H_{, x x}-H_{, y y}\right) \tilde{\theta}_{, x y} .
\end{aligned}
$$

For simplicity, we choose

$$
\tilde{\theta}=a(u) x+b(u) y+c(u),
$$

and Eq. (94) reduces to the Poisson equation

$$
\Delta H=f .
$$

The source term $f$ solves a linear first order PDE

$$
b f_{, x}-a f_{, y}-f=0,
$$

whose general solution [assuming $b(u) \neq 0$ ]

$$
f(u, x, y)=e^{x / b(u)} \phi[a(u) x+b(u) y]
$$

contains one arbitrary function $\phi$ of the argument $a(u) x+$ $b(u) y$. We shall assume this function to be non-vanishing so that $R_{a b} \neq 0$. We can now insert Eq. (98) into the Poisson equation and solve for $H(x, y, u)$. We need two boundary conditions to determine $H$ from the Poisson equation [Eq. (96)] and another one to determine the arbitrary function $\phi$ in Eq. (98). Let us then provide an example by assuming that $b(u)<0$ and $\phi$ remains bounded. In this case, we must restrict the range of the coordinates to the half-plane, $0 \leq x<\infty,-\infty<y<\infty$. We impose a boundary condition $H_{0}(u, y):=H(u, 0, y)$ and appropriate fall-off behavior for $|y| \rightarrow \infty$. We then obtain the particular solution

$$
\begin{aligned}
H(u, x, y) & =\frac{1}{\pi} \int_{-\infty}^{\infty} \frac{x H_{0}(u, \eta) d \eta}{x^{2}+(y-\eta)^{2}} \\
& -\frac{1}{4 \pi} \int_{0}^{\infty} \int_{-\infty}^{\infty} e^{-\xi /|b(u)|} \phi[a(u) \xi+b(u) \eta] \\
& \times \ln \left[\frac{(x+\xi)^{2}+(y-\eta)^{2}}{(x-\xi)^{2}+(y-\eta)^{2}}\right] d \xi d \eta
\end{aligned}
$$

where the double integral extends over the half-plane.

The exponential behavior in Eq. (98) is particularly interesting, since it resembles the gravitational wave solutions found in Refs. [8, 17, 18, 20]. Moreover, as $x \rightarrow \pm \infty$ [depending on the sign of $b(u)$ ] the source term in Eq. (96) diverges, indicating a possible instability. Since we were mainly concerned with the existence of solutions we have not attempted to construct solutions for more general $\theta$ than Eq. (95).

\section{Losing a Killing Vector}

From the analysis so far, it is clear that stationary and axisymmetric solutions in CS gravity do not seem 
to be capable of describing physical spinning BHs. The far-field solution has guided us in the direction of loss of axisymmetry, which in essence corresponds to losing the $\left(\partial_{\phi}\right)^{a}$ Killing vector. Analogously we could conceive of losing stationarity instead of axisymmetric by dropping the $\left(\partial_{t}\right)^{a}$ Killing vector. The general idea is then that by losing one Killing vector we gain new undetermined metric components that could allow for a physical spinning $\mathrm{BH}$ solution in CS gravity. However, our attempts have not revealed any interesting exact solution corresponding to a spinning $\mathrm{BH}$, so we confine ourselves to a couple of general remarks.

Spinning BHs that break axisymmetry or stationarity would be radically different from those considered in GR. On the one hand, non-axisymmetric spinning objects would have an intrinsic precession rate that would not allow the identification of an axis of rotation. Such precession would possibly also lead to solutions with more than two non-zero multipole moments, thus violating the nohair theorem. On the other hand, non-stationary spinning objects would unavoidably lead to the emission of gravitational radiation, even if the $\mathrm{BH}$ is isolated. These considerations could be flipped if we take them as predictions of the theory, thus leading to new possible tests of CS gravity. Work along these lines is currently underway. The results of [73] for the Pontryagin constraint may be helpful here.

\section{Adding matter}

The inclusion of matter sources is of relevance in the present context for several reasons. First, the Kerr BH has a distributional energy momentum tensor 74], so we need not set the stress-energy tensor strictly to zero to construct a Kerr-like solution. Second, in Ref. [16] the Pontryagin-term in the action arises from matter currents, so the inclusion of the latter would actually be mandatory within that framework.

Two conceptually different approaches are possible to the problem of finding exact solutions of the modified field equations in the presence of matter. These approaches essentially depend on whether we require the energy-momentum tensor to be covariantly conserved, $\nabla_{a} T^{a b}=0$, or not. If this tensor is conserved, then the Pontryagin constraint must be satisfied and the Kerr $\mathrm{BH}$ cannot be a solution. Basically, this route leads to only a slight generalization of the discussion presented so far, with solutions of class $\mathcal{P}$,

$$
R_{a b}-\frac{1}{2} g_{a b} R=8 \pi T_{a b}, \quad C_{a b}=0
$$

and solutions of class $\mathcal{C S} \backslash \mathcal{P}$ that solve Eq. (15). Relaxing covariant conservation of the stress-energy tensor, we can promote the Kerr $\mathrm{BH}$ to a solution of the modified field equations, provided that

$$
R_{a b}-\frac{1}{2} g_{a b} R=8 \pi T_{a b}^{\mathrm{dist}}, \quad C_{a b}=8 \pi T_{a b}^{\mathrm{ind}} .
$$

Here $T_{a b}^{\text {dist }}=0$ except for the usual distributional contributions for Kerr [74], while $T_{a b}^{\text {ind }}$ provides the nonconserved matter flux. The induced matter fluxes for the Kerr $\mathrm{BH}$ are given by

$$
\begin{aligned}
& T_{t r}^{\text {ind }}=\frac{a m^{2}}{4 \pi \mu \Sigma^{5} \Delta} \cos \Theta\left(r^{2}-a^{2} \cos ^{2} \Theta\right) \\
& \quad\left[a^{2} \cos ^{2} \Theta\left(11 r^{2}-a^{2}\right)+r^{2}\left(3 r^{2}-9 a^{2}\right)\right], \\
& T_{t \Theta}^{\text {ind }}=-\frac{a m^{2} r}{4 \pi \mu \Sigma^{5}} \sin \Theta \\
& \quad\left[3 r^{4}-12 r^{2} a^{2} \cos ^{2} \Theta+a^{4} \cos ^{4} \Theta\right], \\
& T_{\phi r}^{\text {ind }}=-\frac{a^{2} m^{2}}{4 \pi \mu \Sigma^{5} \Delta} \sin ^{2} \Theta \cos \Theta\left[\cos ^{4} \Theta a^{4}\left(a^{2}-r^{2}\right)\right. \\
& \left.\quad+\cos ^{2} \Theta r^{2} a^{2}\left(8 a^{2}+12 r^{2}\right)-9 r^{4} a^{2}-3 r^{6}\right], \\
& T_{\phi \Theta}^{\text {ind }}=-a \sin ^{2} \Theta T_{t \Theta}^{\text {ind }} .
\end{aligned}
$$

Of course, with such a method any GR solution can be promoted to a solution of the modified field equations.

The crucial issue here is whether or not the induced matter flux can be regarded as physically acceptable. In order to shed light on this issue, we analyzed if the induced stress-energy given by Eqs. (102)-1105) obeys the energy conditions of GR [75]. Because $T_{a b}^{\text {ind }}$ is always traceless, the strong and weak energy conditions are equivalent and reduce to the statement that $T_{a b}^{\text {ind }} \xi^{a} \xi^{b} \geq 0$ for any timelike vector $\xi^{a}$. This, however, is not the case, as we can show by considering for instance $\xi^{t}=$ $\sqrt{2}, \xi^{r}=1$, which is timelike for sufficiently large $r$ : $\xi^{a} \xi^{b} g_{a b}=-1+6 m / r+\mathcal{O}(m / r)^{2}$. The only relevant component of $T_{a b}^{\text {ind }}$ is given by Eq. (102), but since $T_{t r}^{\text {ind }}$ is proportional to $\cos \Theta$, this quantity is negative in half of the spacetime, and thus the weak energy condition is violated. While this might be tolerated close to the horizon, we stress that this violation arises also in the asymptotic region. This violation is somewhat attenuated by the fall-off behavior of $T_{a b}^{\text {ind }}$, where its components decay at least as $1 / r^{5}$ and the scalar invariant $T_{a b}^{\mathrm{ind}} T^{a b}$ ind as $1 / r^{12}$ as $r \rightarrow \infty$. Thus, if ordinary matter is added then the induced exotic fluxes might not be detectable after all for a far-field observer.

There is another approach capable of circumventing the Pontryagin constraint that also relies on new matter sources. Namely, if the field $\theta$ is considered a dynamical field, instead of an external field, it is natural to study more general actions than Eq. (4) with $S_{\text {mat }}=0$, such as [21]

$$
S=\kappa \int d^{4} x \sqrt{-g}\left(R+\frac{1}{2}(\nabla \theta)^{2}-V(\theta)-\frac{1}{\alpha} \theta * R R\right)
$$

Then the Pontryagin constraint (18) is replaced by

$$
{ }^{*} R R=-\alpha\left[\square \theta+V^{\prime}(\theta)\right]
$$

where $\alpha$ is a constant. This provides a natural generalization of the model considered in our paper. However, it also introduces an amount of arbitrariness, since $V$ is a 
free function and, in fact, more general couplings between $\theta$ and curvature might be considered.

We conclude that allowing GR solutions to be also CS solutions by inducing a stress-energy tensor via Eq. (101) can lead to unphysical energy distributions. In particular, the Kerr solution induces an energy momentum tensor given by Eqs. (102)-(105), which violates all energy conditions, even in the asymptotic region. The alternative approach described above lifts $\theta$ to a genuine dynamical field with a kinetic term and possibly self-interactions, at the cost of introducing an arbitrary potential.

\section{CONCLUSIONS AND DISCUSSION}

No exact solution has yet been found that could possibly represent a spinning BH in CS modified gravity. In particular, the Kerr solution is found to be incompatible with the constraints imposed by the modified field equations. Previously, only perturbative solutions of CS gravity had been considered, which might represent the exterior of a BH. The first study was carried out by Alexander and Yunes [18, 19], who performed a weak-field parameterized post-Newtonian analysis to find a non-axisymmetric Kerr-like solution. This study was later extended by Smith, et. al. 21] to non-point like sources, finding that the Israel junction conditions are effectively modified by the C-tensor. Another study was carried out by Konno, et. al. [33], but this analysis was restricted to a limited class of perturbations that did not allow for the breakage of stationarity or axisymmetry. Within that restricted perturbative framework, a Kerrlike solution was found only for non-canonical choices of $\theta$, concluding that BHs cannot rotate in the modified theory for canonical $\theta$. This conclusion of Konno et. al. is at odds with both the results of Alexander and Yunes and Smith et. al.

In order to address these issues, in the current paper we attempted to determine what replaces the Kerr solution in CS modified gravity. We thus studied exact solutions of the modified theory, comprising spherically symmetric, static-axisymmetric, and stationaryaxisymmetric vacuum configurations, as well as some generalizations thereof.

We began our analysis in Sec. IIA by considering the $\mathrm{CS}$ action in detail and rederiving the equations of motion, together with the resultant surface integral terms. We continued in Sec. IIB by rederiving the Pontryagin constraint from the equations of motion and providing two alternative interpretations of it. One of them [Eq. (21)] is a reality condition on a quadratic curvature invariant of the Weyl tensor, while the other [Eq. (24)] is a null condition on the contraction of the electric and magnetic parts of the Weyl tensor. Before considering specific line elements, in Sec. IIC we classified all solutions into two groups: GR-type (class $\mathcal{P}$ ), which contains solutions of both the vacuum Einstein equations and the modified field equations; non-GR type (class $\mathcal{C S} \backslash \mathcal{P}$ ), which contains solutions of CS gravity that are not solutions of the vacuum Einstein equations (cf. Fig. 1).

After these general considerations, we began a systematic study of line elements, starting with general spherically symmetric metrics in Sec. III]. This class of line elements [Eq. (33)] is particularly important since it contains the Schwarzschild, Friedmann-Robertson-Walker and Reissner-Nordström solutions. We showed that, for the canonical choice of the CS scalar field [Eq. (27)] and more general choices [Eq. (34)], the modified field equations decouple and any possible solution is forced to be of class $\mathcal{P}$.

We continued in Sec. IV with an analysis of static and axisymmetric metrics [Eq. (37)]. We showed that, for the canonical choice of the CS scalar field and more general choices [Eq. (55)], the modified equations decouple once more. We also showed that any static and axisymmetric line element is forced to become spatially conformally flat, provided the field equations decouple. Exploiting this result, we found three different solutions [Eqs. (26), (53) and (54)], only one of which was physically relevant, namely the Schwarzschild solution. For the most general CS scalar field, however, the field equations do not decouple, but we have shown that fields with such generality do not seem to allow for a solution to the field equations apart from trivial ones. Thus, we may conclude that CS gravity does not allow for static and axisymmetric solutions, apart from flat space, the Schwarzschild solution and two additional (unphysical) solutions, irrespective of the choice of the CS scalar field.

Static line elements then gave way to the central point of this paper: stationary and axisymmetric solutions of CS gravity, discussed in Sec. V As in the previous cases, we showed that, for the canonical choice of the CS scalar field and slightly more general choices [Eq. [69)], the field equations again decouple. In this case, however, the Pontryagin constraint does not hold automatically and we used it to constrain the class of possible metric functions, cf. appendix B. In essence, the decoupling requires not only that solutions must obey the Einstein equations, but also the fulfillment of additional constraints (cf. Ref. 68]), which leads to an overdetermined system of PDEs. Therefore, we concluded that non-trivial stationary and axisymmetric solutions do not seem to exist for canonical CS fields.

When a completely generic CS scalar field is considered, the modified field equations do not decouple and solutions are not easy to find, even with the simplifications derived from the Pontryagin constraint. However, generic CS fields increase the degrees of freedom of the problem and thus might allow for stationary and axisymmetric solutions. We proved this statement by providing an example in Sec. $\mathrm{VB}$ through a sub-class of stationary and axisymmetric metrics [Eq. (70)], belonging to the van Stockum class. In that case, we showed that the only possible CS field compatible with the field equations excludes the canonical choice. Moreover, we found both, non-flat solutions of class $\mathcal{P}$ [Eq. (73)] as well as non-flat 
solutions of class $\mathcal{C S} \backslash \mathcal{P}$ [Eq. (74)] To the best of our knowledge, this is the first time an exact solution in CS modified gravity is constructed that is not also a solution of GR. One of these solutions [Eq. (74)] represents mathematical BHs, in the sense that although they exhibit a Killing horizon, they are not physically relevant, because the Killing vector generating the 'axial' symmetry is light-like and closed timelike curves arise. We concluded that it is unlikely that stationary, axisymmetric solutions exist that represent a spinning physical $\mathrm{BH}$.

Finally, in Sec.VI we considered the possibility of constructing solutions beyond the set of stationary and axisymmetric spacetimes. We began in Sec. VIA by considering CS scalar fields whose velocity is a Killing vector of the spacetime and found that the only interesting case arises if that vector is null. Naturally, such considerations led to exact gravitational shock-wave spacetimes [Eq. (80)]. Within this pp-wave scenario, in Sec. VIB we constructed a generating method through which any pp-wave solution of GR can be lifted to a solution of CS modified gravity with an appropriate choice of the CS scalar field. We also built a solution of class $\mathcal{C S} \backslash \mathcal{P}$ [Eq. (99)] that is not a GR pp-wave solution but does satisfy the CS modified field equations.

Through this detailed study of solutions in CS gravity we have ascertained that at least two different limits of the Kerr $\mathrm{BH}$ are solutions to the modified field equations, even though the Kerr BH is not: the Schwarzschild limit and the Aichelburg-Sexl limit. The former was already known to be a solution to the CS modified field equations, but the latter, which includes ultrarelativistically boosted BHs, was not. The existence of these solutions concurs with the naive expectations expressed at the end of Sec. IIB. Moreover, such expectations, together with the non-axisymmetric far-field solution, point to the existence of a physical spinning BH solution in CS gravity, provided spacetimes with only one Killing vector are considered. We addressed this possibility briefly in Sec.VIC, but unfortunately such spacetimes are so general that the modified field equations become prohibitively difficult, even with the use of symbolic manipulation software.

Other possibilities of bypassing the Pontryagin constraint were discussed in Sec. VID since this constraint is in essence responsible for the absence of interesting stationary and axisymmetric solutions. First, we stated that obviously any (GR or non-GR) solution formally can be lifted to a solution of the modified field equations by allowing for arbitrary matter sources, and we demonstrated the nature of these matter sources for the Kerr $\mathrm{BH}$. We found that the induced energy momentum tensor [Eqs. (102)-(105)] is exotic even in the asymptotic region, but drops off rapidly with the radial coordinate. Second, we mentioned the possibility that the CS scalar field $\theta$ might acquire a kinetic term and self-interactions. In this case, the Pontryagin constraint ceases to hold and is replaced by a dynamical condition [Eq. (107)], relating the gravitational instanton density to the (generalized) Klein-Gordon operator acting on $\theta$.
We now conclude with a list of possible directions for future research to which our current work may provide the basis.

- The number of physical degrees of freedom in CS modified gravity is not known yet. Various considerations appear to lead to contradictory expectations. On the one hand, the appearance of higher order derivatives in the action [Eq. (1)] suggests that additional degrees of freedom should emerge. On the other hand, the appearance of an additional constraint [Eq. (67)] suggests that fewer degrees of freedom should arise. Actually, the linearization procedure suggests that these competing effects cancel each other and that there are two polarizations of gravitons, just like in GR, albeit with properties that differ from GR [8, 20].

- The role of boundary terms induced in CS gravity for $\mathrm{BH}$ thermodynamics could be investigated more thoroughly [6]. Also here general considerations lead to contradictory expectations. On the one hand, new boundary terms that arise in CS gravity [Eq. (14)] differ qualitatively from those that arise in GR or in scalar tensor theories. Such boundary terms suggest modifications of $\mathrm{BH}$ thermodynamics, even for solutions whose line elements coincide with GR solutions, like the Schwarzschild spacetime. On the other hand, the Pontryagin constraint eliminates the CS contribution [Eq. (1)] to the on-shell action, which suggests that $\mathrm{BH}$ thermodynamics is left unchanged, at least in the classical approximation.

- Both previous issues can be addressed by a thorough Hamiltonian analysis, which is also of interest by itself and for exhibiting the canonical structure as well as the classical constraint algebra. Such a study would also be useful for numerical evolutions of BH binary spacetimes in CS gravity, which is currently being carried out [77].

- While our discussion of stationary and axisymmetric solutions was quite comprehensive, a few issues are still open, which may be an interesting topic for mathematical relativists. For instance, while we were able to provide a proof that there are only three types of solutions for static and axisymmetric spacetimes (with the canonical choice for the CS scalar field), we could only provide good evidence, but no mathematical proof, that no further solutions exist for spacetimes that are stationary and axisymmetric.

- Combining the evidence found in this paper with the far field solutions found previously, we concluded that spinning BHs should break either stationarity or axisymmetry (or both) in CS modified gravity. Perturbations away from axisymmetry were neglected in [33], although non-axisymmetric 
solutions can still represent spinning $\mathrm{BHs}$, albeit with an inherent precession induced by the CS modification. Therefore, future work could focus on finding exact spacetimes with a smaller amount of symmetries.

- A manageable implementation of the Pontryagin constraint could be useful in many CS gravity applications. The brute force methods that led us to the formulas in appendix B will render any generalization unintelligible. The considerations presented in Ref. [73] provide such an implementation, but it has not been exploited so far in the construction of explicit solutions.

- Far-field solutions of CS gravity that break stationarity could also be studied. These solutions could then be used as tests of the modified theory, through comparisons with gravitational-wave and astrophysical observations [78].

- Perhaps it is feasible to apply the method of matched asymptotic expansion for caged $\mathrm{BHs}$ 79, 80] to the construction of spinning $\mathrm{BH}$ solutions in the present context. To this end, one would need an asymptotic expansion and a near horizon expansion of that $\mathrm{BH}$. The former exists already, so it remains to construct the latter and perform the asymptotic matching.

- Finally, it is worthwhile to consider not just vacuum solutions, but also solutions with matter sources, as outlined briefly in Sec. VID.

Certainly the range of issues that can be addressed has been extended in a non-negligible way. Only through a better understanding of the consequences and predictions of CS gravity will we be able to determine the viability of the modified theory.

\section{Acknowledgments}

We are grateful to Stephon Alexander and Roman Jackiw for encouraging us to study this problem in the first place and for enlightening discussions. We would also like to thank Abhay Ashtekar, Henriette Elvang, Alexander Hariton, Scott Hughes, Ralf Lehnert, Ben Owen, Richard O'Shaughnessy, Carlos Sopuerta, Max Tegmark and Richard Woodard for discussions and comments. Most of our calculations used the computer algebra systems MAPLE v.11 in combination with the GRTensorII package 51.

DG is supported in part by funds provided by the U.S. Department of Energy (DoE) under the cooperative research agreement DEFG02-05ER41360. DG has been supported by the project MC-OIF 021421 of the European Commission under the Sixth EU Framework Programme for Research and Technological Development (FP6).
NY acknowledges the support of the Center for Gravitational Wave Physics funded by the National Science Foundation under Cooperative Agreement PHY-0114375 and support from NSF grant PHY-05-55-628.

\section{APPENDIX A: PROOF OF ${ }^{*} R R={ }^{*} C C$}

The equality

$$
{ }^{*} R R={ }^{*} C C
$$

relates the Pontryagin term expressed as in Eq. (5) to the Weyl tensor

$$
C^{a b}{ }_{c d}:=R^{a b}{ }_{c d}-2 \delta_{[c}^{[a} R_{d]}^{b]}+\frac{1}{3} \delta_{[c}^{a} \delta_{d]}^{b} R
$$

and its dual

$$
{ }^{*} C^{a}{ }_{b}{ }^{c d}:=\frac{1}{2} \epsilon^{c d e f} C^{a}{ }_{b e f}
$$

Equation A1 is quite simple to prove, but not entirely obvious. Indeed, we were not able to find it in any of the standard textbooks, review articles or papers on CS modified gravity. Therefore, we provide here a proof by straightforward calculation.

Proof. Let us begin by inserting the definitions (5), (A2) and (A3) into (A1),

$$
{ }^{*} R R={ }^{*} R_{b}^{a}{ }^{c d} R^{b}{ }_{a c d}={ }^{*} C^{a}{ }_{b}{ }^{c d} C^{b}{ }_{a c d}+\Delta .
$$

where $\Delta$ is precisely the violation of Eq. (A1). Thus, if we can show that $\Delta$ vanishes in Eq. (A4) we have proven Eq. (A1). The quantity $\Delta$ contains eight terms. Four of them are linear in the Weyl tensor. Two of these terms are proportional to $C_{c d e f}$ and two are proportional to $C_{c d e b}$. Since

$$
\epsilon^{c d e f} C_{c d e f}=\epsilon^{c d e f} C_{c d e b}=0
$$

these terms vanish. Each of the remaining four terms contains at least two Kronecker $\delta$. These terms always lead to a contraction of the Levi-Civita tensor, e.g. of the form $\epsilon^{c d}{ }_{c}^{f}=0$. Therefore, also these four terms vanish and establish

$$
\Delta=0
$$




\section{APPENDIX B: PONTRYAGIN CONSTRAINT}

For the line element Eq. (66) the Pontryagin constraint Eq. (18) is given by $\left(\bar{w}:=e^{-2 U} w\right)$

$$
0=A_{0} \bar{w}+A_{1} \bar{w}^{3}+A_{2} \bar{w}_{, \rho}+A_{3} \bar{w}^{2} \bar{w}_{, \rho}+A_{4} \bar{w}_{, \rho}^{2}+A_{5} \bar{w}_{, z}+A_{6} \bar{w}^{2} \bar{w}_{, z}+A_{7} \bar{w}_{, z}^{2}+A_{8} \bar{w}_{, \rho} \bar{w}_{, z}+A_{9} \bar{w}_{, \rho z}+A_{10}\left(\bar{w}_{, \rho \rho}-\bar{w}_{, z z}\right)
$$

with

$$
\begin{aligned}
& A_{0}=-\rho^{2} A_{1}+2 \rho^{2}\left(2 U_{, \rho z} k_{, \rho}-\left(U_{, \rho \rho}-U_{, z z}\right) k_{, z}-2 U_{, \rho \rho} U_{, z}+2 U_{, \rho z} U_{, \rho}+4 U_{, z}\left(U_{, \rho}^{2}+U_{, z}^{2}\right)+U_{, z}\left(k_{, \rho \rho}+k_{, z z}\right)\right. \\
& \left.+8 U_{, \rho} U_{, z} k_{, \rho}-6 U_{, \rho}^{2} k_{, z}+2 U_{, z}^{2} k_{, z}-2 U_{, z}\left(k_{, \rho}^{2}+k_{, z}^{2}\right)\right)+2 \rho\left(U_{, \rho} k_{, z}-U_{, z} k_{, \rho}+2 U_{, z} U_{, \rho}\right) \\
& A_{1}=8 \rho\left(U_{, \rho} U_{, z}\left(U_{z z}-U_{\rho \rho}\right)+U_{, \rho z}\left(U_{, \rho}^{2}-U_{, z}^{2}\right)-U_{, \rho}^{3} k_{, z}+U_{, z}^{3} k_{, \rho}+U_{, \rho} U_{, z}\left(U_{, \rho} k_{, \rho}-U_{, z} k_{, z}\right)\right)+8 U_{, z}\left(U_{, \rho}^{2}+U_{, z}^{2}\right) \\
& A_{2}=2 \rho^{3}\left(2 U_{, z z} U_{, z}+2 U_{, \rho z} U_{, \rho}+4 U_{, \rho}^{2} U_{, z}+4 U_{, z}^{3}+\left(U_{, \rho \rho}-U_{, z z}\right) k_{, z}-U_{, z}\left(k_{, \rho \rho}+k_{, z z}\right)-2 U_{, \rho z} k_{, \rho}-4 U_{, \rho} U_{, z} k_{, \rho}\right. \\
& \left.+2 U_{, z} k_{, \rho}^{2}+2 U_{, z} k_{, z}^{2}-4 U_{, z}^{2} k_{, z}\right)-2 \rho^{2}\left(U_{, \rho z}+4 U_{, z} U_{, \rho}-2 U_{, \rho} k_{, z}\right)-\rho k_{, z} \\
& A_{3}=4 \rho\left(3 U_{, \rho}^{2} k_{, z}+U_{, z}^{2} k_{, z}-2 U_{, \rho} U_{, z} k_{, \rho}-4 U_{, \rho}^{2} U_{, z}+\left(U_{, \rho \rho}-U_{, z z}\right) U_{, z}-2 U_{, \rho} U_{, \rho z}-4 U_{, z}^{3}\right)-8 U_{, \rho} U_{, z} \\
& A_{4}=2 \bar{w}\left(\rho U_{, z} k_{, \rho}-3 \rho U_{, \rho} k_{, z}+U_{, z}+6 \rho U_{, \rho} U_{, z}+\rho U_{, \rho z}\right)-\bar{w}_{, \rho} \rho\left(2 U_{, z}-k_{, z}\right) \\
& A_{5}=2 \rho^{3}\left(-2 U_{, \rho \rho} U_{, \rho}-2 U_{, \rho z} U_{, z}-4 U_{, z}^{2} U_{, \rho}-4 U_{, \rho}^{3}+\left(U_{, \rho \rho}-U_{, z z}\right) k_{, \rho}+U_{, \rho}\left(k_{, z z}+k_{, \rho \rho}\right)+2 U_{, \rho z} k_{, z}+4 U_{, z} U_{, \rho} k_{, z}\right. \\
& \left.-2 U_{, \rho}\left(k_{, \rho}^{2}+k_{, z}^{2}\right)+4 U_{, \rho}^{2} k_{, \rho}\right)+2 \rho^{2}\left(U_{, \rho \rho}+4 U_{, \rho}^{2}-2 U_{, \rho} k_{, \rho}\right)+\rho^{2}\left(2 k_{, \rho}^{2}+2 k_{, z}^{2}-k_{, \rho \rho}-k_{, z z}\right)-\rho\left(2 U_{, \rho}-k_{, \rho}\right) \\
& A_{6}=4 \rho U_{, \rho}\left(U_{, \rho \rho}-U_{, z z}\right)+8 \rho U_{, z} U_{, \rho z}-4 U_{, \rho}^{2}-12 U_{, z}^{2}+4 \rho\left(4 U_{, \rho}\left(U_{, \rho}^{2}+U_{, z}^{2}\right)-U_{, \rho}^{2} k_{, \rho}+2 U_{, z} U_{, \rho} k_{, z}-3 U_{, z}^{2} k_{, \rho}\right) \\
& A_{7}=\bar{w}\left(6 U_{, z}-2 \rho U_{, \rho z}-12 \rho U_{, z} U_{, \rho}-2 \rho U_{, \rho} k_{, z}+6 \rho U_{, z} k_{, \rho}\right)+\bar{w}_{, z} \rho\left(2 U_{, \rho}-k_{, \rho}\right)-\bar{w}_{, z} \\
& A_{8}=2 \bar{w} \rho\left(U_{, z z}-U_{, \rho \rho}+6 U_{, z}^{2}-6 U_{, \rho}^{2}-2 U_{, z} k_{, z}+2 U_{, \rho} k_{, \rho}\right)+4 \bar{w} U_{, \rho} \\
& +\bar{w}_{, \rho} \rho\left(2 U_{, \rho}-k_{, \rho}\right)-\bar{w}_{, \rho}-\bar{w}_{, z} \rho\left(2 U_{, z}-k_{, z}\right) \\
& A_{9}=2 \rho^{3}\left(U_{, z z}-U_{, \rho \rho}+2 U_{, z}^{2}-2 U_{, \rho}^{2}+2 U_{, \rho} k_{, \rho}-2 U_{, z} k_{, z}\right)-2 \rho^{2} k_{, \rho}-4 \bar{w}^{2} \rho\left(U_{, \rho}^{2}-U_{, z}^{2}\right) \\
& +4 \bar{w} \rho\left(\bar{w}_{, \rho} U_{, \rho}-\bar{w}_{, z} U_{, z}\right)-\left(\bar{w}_{, \rho}^{2}-\bar{w}_{, z}^{2}\right) \rho \\
& A_{10}=2 \rho^{3}\left(U_{, \rho z}+2 U_{, \rho} U_{, z}-U_{, \rho} k_{, z}-U_{, z} k_{, \rho}\right)+\rho^{2} k_{, z}+4 \bar{w}^{2} \rho U_{, \rho} U_{, z}-2 \bar{w} \bar{w}_{, \rho} \rho U_{, z}-2 \bar{w} \bar{w}_{, z} \rho U_{, \rho}+\bar{w}_{, \rho} \bar{w}_{, z} \rho
\end{aligned}
$$

[1] C. M. Will, Living Rev. Relativity 9, 3 (2005), grqc/0510072.

[2] A. G. Riess et al. (Supernova Search Team), Astron. J. 116, 1009 (1998), astro-ph/9805201.

[3] S. Perlmutter et al. (Supernova Cosmology Project), Astrophys. J. 517, 565 (1999), arXiv:astro-ph/9812133.

[4] M. Tegmark et al., Phys. Rev. D74, 123507 (2006), astro-ph/0608632.

[5] P. Jordan, Z. Phys. 157, 112 (1959).

[6] C. Brans and R. H. Dicke, Phys. Rev. 124, 925 (1961).

[7] R. P. Woodard (2006), astro-ph/0601672.

[8] R. Jackiw and S. Y. Pi, Phys. Rev. D68, 104012 (2003), gr-qc/0308071.

[9] L. Alvarez-Gaume and E. Witten, Nucl. Phys. B234, 269 (1984).

[10] J. S. Bell and R. Jackiw, Nuovo Cim. A60, 47 (1969).

[11] M. B. Green, J. H. Schwarz, and E. Witten, Superstring Theory. Vol. 2: Loop Amplitides, Anomalies and Phenomenology (Cambridge University Press (Cambridge Monographs On Mathematical Physics), Cambridge, Uk, 1987).

[12] S. H. S. Alexander and J. Gates, S. James, JCAP 0606, 018 (2006), hep-th/0409014.
[13] A. Lue, L.-M. Wang, and M. Kamionkowski, Phys. Rev. Lett. 83, 1506 (1999), astro-ph/9812088.

[14] M. Li, J.-Q. Xia, H. Li, and X. Zhang (2006), hep$\mathrm{ph} / 0611192$.

[15] S. H. S. Alexander (2006), hep-th/0601034.

[16] S. H. S. Alexander, M. E. Peskin, and M. M. SheikJabbari, Phys. Rev. Lett. 96, 081301 (2006), hepth/0403069.

[17] S. Alexander and J. Martin, Phys. Rev. D71, 063526 (2005), hep-th/0410230.

[18] S. Alexander and N. Yunes (2007), hep-th/0703265.

[19] S. Alexander and N. Yunes, Phys. Rev. D75, 124022 (2007), arXiv:0704.0299 [hep-th].

[20] S. Alexander, L. S. Finn, and N. Yunes, in progress (2007).

[21] T. L. Smith, A. L. Erickcek, R. R. Caldwell, and M. Kamionkowski (2007), arXiv:0708.0001 [astro-ph].

[22] D. Guarrera and A. J. Hariton, Phys. Rev. D76, 044011 (2007), gr-qc/0702029.

[23] V. A. Kostelecky, Phys. Rev. D69, 105009 (2004), hepth/0312310.

[24] T. Mariz, J. R. Nascimento, E. Passos, and R. F. Ribeiro, Phys. Rev. D70, 024014 (2004), hep- 
th/0403205.

[25] R. Bluhm and V. A. Kostelecky, Phys. Rev. D71, 065008 (2005), hep-th/0412320.

[26] C. Eling, T. Jacobson, and D. Mattingly (2004), grqc/0410001.

[27] S. H. S. Alexander, M. E. Peskin, and M. M. SheikhJabbari, Phys. Rev. Lett. 96, 081301 (2006), hepth/0403069.

[28] D. H. Lyth, C. Quimbay, and Y. Rodriguez, JHEP 03, 016 (2005), hep-th/0501153.

[29] D. Mattingly, Living Rev. Rel. 8, 5 (2005), grqc/0502097.

[30] R. Lehnert (2006), gr-qc/0602073.

[31] A. J. Hariton and R. Lehnert, Phys. Lett. A367, 11 (2007), hep-th/0612167.

[32] S. H. Alexander, M. E. Peskin, and M. M. SheikhJabbari (2007), hep-ph/0701139.

[33] K. Konno, T. Matsuyama, and S. Tanda, Phys. Rev. D76, 024009 (2007), arXiv:0706.3080 [gr-qc].

[34] W. Fischler and S. Paban (2007), arXiv:0708.3828 [hepth].

[35] B. Tekin (2007), arXiv:0710.2528 [gr-qc].

[36] R. H. Brandenberger and C. Vafa, Nucl. Phys. B316, 391 (1989).

[37] A. A. Tseytlin and C. Vafa, Nucl. Phys. B372, 443 (1992), hep-th/9109048.

[38] A. Nayeri, R. H. Brandenberger, and C. Vafa, Phys. Rev. Lett. 97, 021302 (2006), hep-th/0511140.

[39] C.-Y. Sun and D.-H. Zhang (2006), hep-th/0611101.

[40] D. H. Wesley, P. J. Steinhardt, and N. Turok, Phys. Rev. D72, 063513 (2005), hep-th/0502108.

[41] S. Alexander, R. H. Brandenberger, and D. Easson, Phys. Rev. D62, 103509 (2000), hep-th/0005212.

[42] R. Brandenberger, D. A. Easson, and D. Kimberly, Nucl. Phys. B623, 421 (2002), hep-th/0109165.

[43] T. Battefeld and S. Watson, Rev. Mod. Phys. 78, 435 (2006), hep-th/0510022.

[44] R. H. Brandenberger, A. Nayeri, S. P. Patil, and C. Vafa (2006), hep-th/0608121.

[45] R. Brandenberger (2007), hep-th/0702001.

[46] P. Brax, C. van de Bruck, and A.-C. Davis, Rept. Prog. Phys. 67, 2183 (2004), hep-th/0404011.

[47] L. Randall and R. Sundrum, Phys. Rev. Lett. 83, 3370 (1999), hep-ph/9905221.

[48] L. Randall and R. Sundrum, Phys. Rev. Lett. 83, 4690 (1999), hep-th/9906064.

[49] B. Aschenbach (2007), arXiv:0710.3454 [astro-ph].

[50] C. W. Misner, K. Thorne, and J. A. Wheeler, Gravitation (W. H. Freeman \& Co., San Francisco, 1973).

[51] GRTensorII, this is a package which runs within Maple but distinct from packages distributed with Maple. It is distributed freely on the World-Wide-Web from the address: http://grtensor.org.

[52] J. W. York, Jr., Phys. Rev. Lett. 28, 1082 (1972).

[53] G. W. Gibbons and S. W. Hawking, Phys. Rev. D15, 2752 (1977).

[54] R. Penrose and W. Rindler, Spinors and Space-Time II (Cambridge University Press, 1986).

[55] H. Stephani, D. Kramer, M. MacCallum, C. Hoenselaers, and E. Herlt, Exact solutions of Einstein's field equations (Cambridge University Press, 2003).

[56] N. Yunes and J. A. Gonzalez, Phys. Rev. D73, 024010 (2006), gr-qc/0510076.

[57] A. Buonanno, G. B. Cook, and F. Pretorius, Phys. Rev.
D75, 124018 (2007), gr-qc/0610122.

[58] C. Cherubini, D. Bini, S. Capozziello, and R. Ruffini, Int. J. Mod. Phys. D11, 827 (2002), gr-qc/0302095.

[59] E. Poisson, A relativist's toolkit (Cambrdige, 2004).

[60] L. Blanchet, Living Rev. Rel. 9, 4 (2006), and references therein, gr-qc/0202016.

[61] R. Lehnert, private communication.

[62] H. Balasin, C. G. Boehmer, and D. Grumiller, Gen. Rel. Grav. 37, 1435 (2005), gr-qc/0412098.

[63] D. Grumiller, W. Kummer, and D. V. Vassilevich, Phys. Rept. 369, 327 (2002), hep-th/0204253.

[64] R. M. Wald, General Relativity (The University of Chicago Press, 1984).

[65] J. Chazy, Bull. Soc. Math. France 52, 17 (1924).

[66] H. E. J. Curzon, Proc. London Math. Soc. 23, 477 (1924).

[67] B. Lukács and Z. Perjés, Phys. Lett. A88, 267 (1982).

[68] http://www.gravity.psu.edu/ yunes/public.html The Maple scripts that generate these quantities are available upon request.

[69] P. Jordan, J. Ehlers, and W. Kundt, Akad. Wiss. Lit. (Mainz) Abhandl. Math.-Nat. Kl. 2, 21 (1960).

[70] P. C. Aichelburg and R. U. Sexl, Gen. Rel. Grav. 2, 303 (1971).

[71] C. O. Lousto and N. G. Sanchez, Nucl. Phys. B383, 377 (1992).

[72] H. Balasin and H. Nachbagauer, Class. Quant. Grav. 12, 707 (1995), gr-qc/9405053.

[73] D. Grumiller and R. Jackiw, arXiv:0711.0181 [math-ph].

[74] H. Balasin and H. Nachbagauer, Class. Quant. Grav. 11, 1453 (1994), gr-qc/9312028.

[75] S. Hawking and G. Ellis, The Large Scale Structure of Space-Time (Cambridge University Press, 1973).

[76] S. Alexander, D. Grumiller, and N. Yunes, in progress (2007).

[77] S. Wood, in preparation.

[78] N. Yunes, in preparation.

[79] D. Gorbonos and B. Kol, JHEP 06, 053 (2004), hepth/0406002.

[80] D. Gorbonos and B. Kol, Class. Quant. Grav. 22, 3935 (2005), hep-th/0505009.

[81] J. Z. Simon, Phys. Rev. D41, 3720 (1990).

[82] J. Z. Simon, Phys. Rev. D43, 3308 (1991).

[83] A. Garcia, F. W. Hehl, C. Heinicke, and A. Macias, Class. Quant. Grav. 21, 1099 (2004), gr-qc/0309008.

[84] T. Regge and J. A. Wheeler, Phys. Rev. 108, 1063 (1957).

[85] C. Sopuerta and N. Yunes (2007), in progress.

[86] A. Matte, Ca. J. Math. 5, 1 (1953).

[87] If one considers the classical solutions of the nonmodified theory (without higher derivatives) and regards additional terms as loop corrections, then no stability issues arise. In that case, additional (unstable) solutions must be considered spurious [81, 82].

[88] The conclusion that stationary and axisymmetric solutions to the modified field equations do not exist is in agreement with 33. However, this fact does not lead to the conclusion that spinning BHs cannot exist in the modified theory, as implied in [33]. In fact, an approximate solution (albeit not axisymmetric) that can represent a spinning $\mathrm{BH}$ in $\mathrm{CS}$ gravity has already been found in the far-field [19, 21].

[89] There is a relative sign difference in the CS correction to the action compared to [8]. This minus sign is included 
in order to obtain the same equations of motion as in [8], correcting a minor typo.

[90] We prefer to work with tensors rather than with tensor densities in this paper, so some expressions might appear to differ by factors of $\sqrt{-g}$ from [8].

[91] If $\nabla_{a} K^{a}$ is converted into $1 / \sqrt{g} \partial_{a}\left(\sqrt{g} K^{a}\right)$ the results (2.4) and (2.5) of [8] are recovered.

[92] In the original work of [8], this tensor was called 'Cotton tensor' because it shares similarities with the 3dimensional Cotton-tensor. However, the notion of a higher-dimensional Cotton tensor already exists [83] and differs from the definition of $C_{a b}$, which is why we refer to Eq. (12) as a "C-tensor".

[93] The quantity $v_{a}$ is sometimes referred to as an embedding coordinate since it embeds the 3-dimensional CS theory into a 4-dimensional spacetime.

[94] When $v_{a}=0$ then $\theta$ is constant and the Pontryagin term becomes a topological term not contributing to the field equations.

[95] One can show that Eq. (24) is related to the vanishing of certain derivatives of the Regge-Wheeler function in the Regge-Wheeler [84] decomposition of the metric perturbation. We are currently studying how such a condition impacts the ringing of CS BHs elsewhere 85]. The equivalence between Eqs. (21) and (24) was shown for the first time in [86].

[96] Actually there is a third limit, $\boldsymbol{E}=0$, which is either trivial (if also $\boldsymbol{B}$ vanishes) or a magnetic monopole.

[97] The persistence of Birkhoff's theorem in CS modified gravity was first proved by one of us (NY) in collaboration with C. Sopuerta 85].

[98] Similar conclusions hold for the Kerr-Newman and Kerr-NUT spacetimes.

[99] We were able to find additional solutions with Maple, but upon imposing the field equations they reduced to previously studied or trivial spacetimes.

[100] There is a straightforward generalization of Eq. (74), namely $\Omega=c+d / \rho^{\alpha}, \theta=\beta \sqrt{\rho} z$, where $\alpha$ is an arbitrary constant and $\beta=8 \alpha /\left(8 \alpha^{2}+2 \alpha+3\right)$.

[101] We attempted to solve the modified field equations with a line element equal to Kerr plus a stationary and axisymmetric contribution and an arbitrary CS scalar field. No solution could be found exactly, while perturbatively such an Ansatz leads to non-asymptotically flat solutions 33. 